\title{
Systematic mapping of rRNA 2'-O methylation during frog development and involvement of the methyltransferase Fibrillarin in eye and craniofacial development in Xenopus laevis
}

\author{
Jonathan Delhermite ${ }^{1}$, Lionel Tafforeau $\oplus^{1,2}$, Sunny Sharma $\oplus^{1}$, Virginie Marchand $\oplus^{3}$, \\ Ludivine Wacheul ${ }^{1}$, Ruben Lattuca ${ }^{4}$, Simon Desiderio $\mathbb{D}^{5}$, Yuri Motorin $\mathbb{D}^{3}$, \\ Eric Bellefroid ${ }^{5}$, Denis L. J. Lafontaine ${ }^{1 *}$ \\ 1 RNA Molecular Biology, Fonds de la Recherche Scientifique (F.R.S./FNRS), Université libre de Bruxelles \\ (ULB), Biopark campus, Gosselies, Belgium, 2 Cell Biology Lab, Research Institute for Biosciences, \\ Université de Mons (UMONS), Mons, Belgium, 3 Université de Lorraine, CNRS, INSERM, EpiRNA-Seq Core \\ Facility, UMS2008/US40 IBSLor and CNRS, UMR7365 IMoPA, Nancy, France, 4 Erasme campus, \\ Université libre de Bruxelles (ULB), Brussels, Belgium, 5 Developmental Biology, Université libre de \\ Bruxelles (ULB), Biopark campus, Gosselies, Belgium \\ *denis.lafontaine@ulb.be
}

\section{OPEN ACCESS}

Citation: Delhermite J, Tafforeau L, Sharma S, Marchand V, Wacheul L, Lattuca R, et al. (2022) Systematic mapping of rRNA 2'-0 methylation during frog development and involvement of the methyltransferase Fibrillarin in eye and craniofacial development in Xenopus laevis. PLoS Genet 18(1): e1010012. https://doi.org/10.1371/journal. pgen.1010012

Editor: Paul A. Trainor, Stowers Institute for Medical Research, UNITED STATES

Received: December 31, 2020

Accepted: December 23, 2021

Published: January 18, 2022

Peer Review History: PLOS recognizes the benefits of transparency in the peer review process; therefore, we enable the publication of all of the content of peer review and author responses alongside final, published articles. The editorial history of this article is available here: https://doi.org/10.1371/journal.pgen.1010012

Copyright: @ 2022 Delhermite et al. This is an open access article distributed under the terms of the Creative Commons Attribution License, which permits unrestricted use, distribution, and reproduction in any medium, provided the original author and source are credited.

Data Availability Statement: All RiboMethSeq files are available from the 'European Nucleotide

\section{Abstract}

Ribosomes are essential nanomachines responsible for protein production. Although ribosomes are present in every living cell, ribosome biogenesis dysfunction diseases, called ribosomopathies, impact particular tissues specifically. Here, we evaluate the importance of the box C/D snoRNA-associated ribosomal RNA methyltransferase fibrillarin (Fbl) in the early embryonic development of Xenopus laevis. We report that in developing embryos, the neural plate, neural crest cells (NCCs), and NCC derivatives are rich in $\mathrm{fb}$ /transcripts. Fbl knockdown leads to striking morphological defects affecting the eyes and craniofacial skeleton, due to lack of NCC survival caused by massive p53-dependent apoptosis. Fbl is required for efficient pre-rRNA processing and 18S rRNA production, which explains the early developmental defects. Using RiboMethSeq, we systematically reinvestigated ribosomal RNA 2'-O methylation in X. laevis, confirming all 89 previously mapped sites and identifying 15 novel putative positions in $18 \mathrm{~S}$ and $28 \mathrm{~S}$ rRNA. Twenty-three positions, including 10 of the new ones, were validated orthogonally by low dNTP primer extension. Bioinformatic screening of the $X$. laevis transcriptome revealed candidate box C/D snoRNAs for all methylated positions. Mapping of 2'-O methylation at six developmental stages in individual embryos indicated a trend towards reduced methylation at specific positions during development. We conclude that fibrillarin knockdown in early Xenopus embryos causes reduced production of functional ribosomal subunits, thus impairing NCC formation and migration. 
Archive' database (accession number PRJEB42253).

Funding: JD and RL were recipients of a FRIA PhD fellowship (F.R.S./FNRS, https://www.frs-fnrs.be/ en/). LT was the recipient of a 'Chargé de Recherches' fellowship from the Fonds de la Recherche Scientifique (F.R.S./FNRS). Research in the Lafontaine laboratory is supported by the Belgian Fonds de la Recherche Scientifique (F.R.S./ FNRS, PDR grant $n^{\circ} T .0144 .20$ ), the European Joint Programmes on Rare Diseases (EJP RD/JTC2019/ PINT-MULTI) 'RiboEurope' and 'DBAGeneCure' (grant $n^{\circ} R .8015 .19$ and $n^{\circ} R .8011 .20$ ), the Université libre de Bruxelles (ULB https://www.ulb. be/en/ulb-homepage), the Région Wallonne (SPW EER https://www.wallonie.be/fr/acteurs-etinstitutions/wallonie/spw-economie-emploirecherche/departement-de-la-recherche-et-dudeveloppement-technologique\#) ('RIBOcancer' FS0 grant n 1810070 and POC n 1880014 ), the Fonds Jean Brachet, the Internationale Brachet Stiftung, and the Epitran COST action (CA16120 https://epitran.eu/). YM was supported by FRCR EpiARN project from Grand Est Region, France. The funders had no role in study design, data collection and analysis, decision to publish, or preparation of the manuscript.

Competing interests: The authors have declared that no competing interests exist.

\section{Author summary}

Ribosomes are essential nanomachines responsible for protein production in all cells. Ribosomopathies are diseases caused by improper ribosome formation due to mutations in ribosomal proteins or ribosome assembly factors. Such diseases primarily affect the brain and blood, and it is unclear how malfunctioning of a process as general as ribosome formation can lead to tissue-specific diseases. Here we have examined how fibrillarin, an enzyme which modifies ribosomal RNA by adding methyl groups at specific sites, affects early embryonic development in the frog Xenopus laevis. We have revealed its importance in the maturation of cells forming an embryonic structure called the neural crest. Fibrillarin depletion leads to reduced eye size and abnormal head shape, reminiscent of other conditions such as Treacher Collins syndrome. Molecularly, the observed phenotypes are explainable by increased p53-dependent programmed cell death triggered by inhibition of certain pre-rRNA processing steps. Our systematic investigation of the ribosomal RNA 2'$\mathrm{O}$ methylation repertoire across development has further revealed hypomodification at a late stage of development, which might play a role in late developmental transitions involving differential translation by compositionally different ribosomes.

\section{Introduction}

Ribosomes are essential nanomachines responsible for protein production in every cell. They are sophisticated ribonucleoprotein particles consisting of two subunits of unequal size with specialized functions in translation. The small subunit, $40 \mathrm{~S}$ in eukaryotes, is responsible for decoding the messenger RNA (mRNA) while the large subunit, 60S, is in charge of joining the amino acids together into polypeptides [1].

Ribosome biogenesis entails numerous reactions, including synthesis of the components (four ribosomal RNAs (rRNAs) and eighty ribosomal proteins in human ribosomes) and their modification, transport, and assembly into functional subunits [2,3]. These reactions are aided by numerous trans-acting factors that interact transiently with the maturing ribosomal subunits [4,5]. Although surveillance mechanisms monitor the faithful production of ribosomal subunits, cells can produce ribosomes that differ in composition and possibly also in function $[6,7]$.

An important source of ribosome diversity is ribosomal RNA modification [8]. There are two prevalent types of rRNA modifications: 2'-O methylation, guided by antisense box C/D snoRNAs carrying the methyltransferase fibrillarin, and pseudouridylation, guided by antisense H/ACA snoRNAs bearing the pseudouridine synthetase DKC1 [9]. Each human ribosome contains $>100$ of each of these two types of modifications on specific residues. These are precisely selected during ribosomal subunit biogenesis by Watson-Crick base-pairing between the snoRNAs and rRNA precursors [10]. In addition, there are a few base modifications, mostly methylation, aminocarboxypropylation, and acetylation [11]. Several rRNA modifications are installed close to important functional sites on the ribosomes, where they are widely assumed to optimize ribosome function $[9,11,12]$. In zebrafish, inhibition of the production of individual box $\mathrm{C} / \mathrm{D}$ snoRNAs with interfering antisense oligonucleotides has revealed that they are important for development [13].

Recent work has indicated that in human cancer cells [14-17] and during development in zebrafish [18] and mouse [19], some of the sites susceptible to be 2'-O methylated are not modified on every ribosome. Cells can thus produce a heterogeneous population of ribosomes potentially displaying differential translational properties, i.e. preferentially translating specific 
mRNAs [20]. Messenger RNAs liable to be differentially translated include molecules harboring particular cis-acting elements such as internal ribosome entry sites [16,21]. Differential translation by compositionally different ribosomes may have important repercussions in normal processes such as cell differentiation and embryogenesis $[22,23]$ and in pathological ones promoting diseases such as cancer and neurodegeneration [21,24,25].

Ribosome biogenesis is a ubiquitous process. Nonetheless, ribosome biogenesis dysfunction impacts more severely particular cells in specific tissues, leading to a diverse set of human syndromes collectively known as ribosomopathies [26,27]. Ribosomopathies are congenital or somatic-tissue-specific diseases resulting from mutations in ribosomal proteins or ribosome biogenesis factors. These lead to a shortage of functional ribosomes and generally to a hypoproliferation phenotype, often accompanied later in life, because of secondary mutations, by an increased susceptibility to developing cancers [28]. The blood and brain are among the prime targets of ribosomopathies, which then manifest as hematopoiesis defects, microcephally, and intellectual disability.

Ribosomopathy-associated symptoms are often deeply rooted in embryonic development. Ribosome biogenesis dysfunction can profoundly impact the development of specific tissues derived or not from the neural crest cells (NCCs). NCCs are multipotent embryonic migratory cells unique to vertebrates. They can specify towards numerous cell types and tissues, including the teeth, peripheral nervous system and eyes, glia, heart, pigmented cells of the skin, and head skeletal structures. Typically, the craniofacial development aberrations associated with the ribosomopathies Treacher-Collins Syndrome and Diamond-Blackfan Anemia (DBA) are caused by neural crest cell maturation problems [26,27]. The bone marrow failure and anemia observed in Shwachman-Diamond Syndrome (SDS), DBA, and dyskeratosis congenita are caused, rather, by defective maturation of non-neural crest cells [27].

The importance of studying ribosome biogenesis factor mutations in animal models is illustrated by multiple examples, including the intellectual disability (ID) recently found to be associated with mutations in METTL5 [29]. This gene encodes a methyltransferase responsible for depositing an $\mathrm{m}^{6} \mathrm{~A}$ mark close to the decoding site of the small ribosomal subunit [30]. In cultured human cells, mettl5 deletion does not cause any obvious cell proliferation defect, but its mutation in the fly leads to severe behavioral problems reminiscent of ID [31]. The zebrafish Danio rerio [32-35], the frogs Xenopus laevis [36,37] and Xenopus tropicalis [38-40], and mouse $[41,42]$ have also proved to be powerful models for approaching ribosomopathies functionally.

Here we have studied the involvement of the box C/D snoRNA-associated methyltransferase fibrillarin in early embryo development in X. laevis. We have revealed its importance in small ribosomal subunit production and NCC survival. We have also investigated systematically by RiboMethSeq the complete 2'-O methylation repertoire of rRNAs in developing frog embryos, confirming previously mapped positions, identifying new ones, and revealing that specific sites are strongly hypomodified at late stages of embryogenesis.

\section{Results}

\section{Expression of $f b l, n c l$, and $u b t f$ during $X$. laevis development}

To investigate the role of the methyltransferase fibrillarin (Fbl) in Xenopus development, we first established its spatiotemporal expression during early embryogenesis. For comparison, we mapped the expression of two additional ribosome assembly factors, namely Ubtf, important in rRNA synthesis, and nucleolin ( $\mathrm{Ncl})$, a late-acting ribosome assembly factor.

We first analyzed the temporal expression of $f b l, n c l$, and $u b t f$ and found all three genes to be expressed maternally (stages 2, 4, and 6), and throughout development, notably after the midblastula transition (MBT, stage 8) (S1 Fig). 
Next, we analyzed the spatial expression of the selected genes by whole-mount in situ hybridization (WISH). We selected five developmental stages corresponding to critical time points during embryogenesis: segmentation (stage 4), gastrulation (stages 10-11), neurulation (stage 18), and early and late organogenesis (stages 25-26, and stage 32). Transcripts of all three genes were detected only at the animal pole of cleaving-stage embryos and at gastrulation (Fig 1A, stage 4 and stages 10-11), suggesting asymmetric distribution of the corresponding maternal transcripts. From neurulation on (stage 18), expression of $f b l, n c l$, and $u b t f$ was observed in the developing neurectoderm. At the tailbud and early tadpole stages (Fig 1A, stages 25-26, and stage 32, respectively), transcripts were detected in the developing eyes, brain (both the midbrain and the hindbrain), and branchial arches. Expression of $n c l$ and $u b t f$ was also observed in the pronephros at these later stages.

We further analyzed the expression of $f b l$, the focus of this work, in animal cap explants. Animal cap cells are ectodermal cells forming the roof of the blastocoele. These cells are pluripotent and specify at that stage to form epidermis. We analyzed $f b l$ expression by RT-qPCR in animal cap explants derived from embryos injected at their animal pole with either noggin mRNA, to induce their differentiation to neural tissue, or a combination of noggin and wnt8 mRNAs, to induce their differentiation to neural crest cells (NCCs) [43]. Expression of $f b l$ appeared similar in neuralized and non-induced control caps, but it was higher in induced NCCs (Fig 1B). Fbl is thus likely to play an important role in NCCs. As controls, the expression of krt12 (encoding the epidermal marker keratin, EpK), sox3 (a neural marker), and slug (an NCCs marker) were determined. As expected, krt 12 was found only in non-induced cells, sox 3 was higher in induced neural caps, and slug was detected only in caps induced to form NCCs (Fig 1B).

We wondered whether the abundance of $f b l$ transcripts in NCCs is an evolutionarily conserved feature. Previously, the distribution of $f b l$ transcripts was studied in the mouse embryo, but the insufficient resolution of the analysis at the time did not allow revealing any specific enrichment in the brain area [44]. To address this, we carefully inspected $f b l$ expression in E14.5 mouse embryos by sectioned in situ hybridization and found abundant $f b l$ transcripts in the retina and the neural-crest-derived mesenchymal cells of the head (Fig 1C). This localization is consistent with that observed in frogs.

In conclusion, $f b l, n c l$, and $u b t f$ appear to be expressed non-uniformly during Xenopus embryonic development, with strikingly stronger expression in neural crest cells and their derivatives. The spatial distribution of $f b l$ transcripts is similar in mouse. Ribosomes are produced in every cell, and $\mathrm{Fbl}$ is essential to ribosome production ([45-47] and see below). Nonetheless, an elevated $f b l$ transcript abundance in particular tissues may be associated with increased $\mathrm{Fbl}$ protein synthesis. If $\mathrm{Fbl}$ is limiting for ribosome synthesis, this increased production might alter the rate or pathway of pre-rRNA packaging, processing or modification.

\section{Effects of $\mathrm{Fbl}, \mathrm{Ncl}$, and Ubtf depletion on $\mathrm{X}$. laevis early development}

To assess the involvement of $\mathrm{Fbl}, \mathrm{Ncl}$, and Ubtf in early embryogenesis, we performed knockdown experiments with antisense morpholinos (MOs) directed against the translation initiation site in their respective mRNAs. Embryos at the two-cell stage were injected unilaterally at the animal pole with $20 \mathrm{ng} \mathrm{MO}$. Injected embryos were fixed at stage 42 and examined for morphological changes (Fig 2A).

Microinjection of a $\mathrm{MO}$ specifically targeting $\mathrm{Fbl}, \mathrm{Ncl}$, or Ubtf mRNAs resulted in a similar and more or less pronounced alteration of the eye. Between 59 and $82.5 \%$ of the injected embryos displayed a reduced eye size accompanied, in $46-59 \%$ of cases, by head asymmetry indicative of craniofacial skeleton alterations (Fig $2 \mathrm{~A}$ and $2 \mathrm{~B}$ ). The area occupied by the retinal 
A

stage 4

stages $10-11$
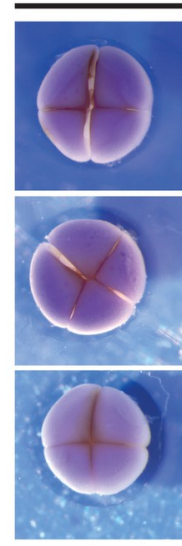

animal
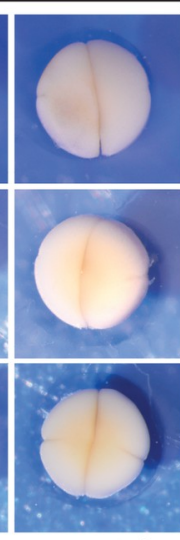

vegetal
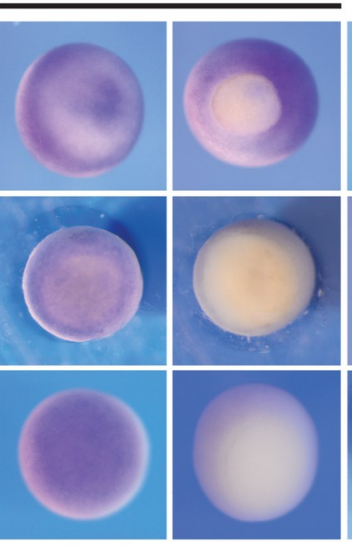

vegetal stage 18

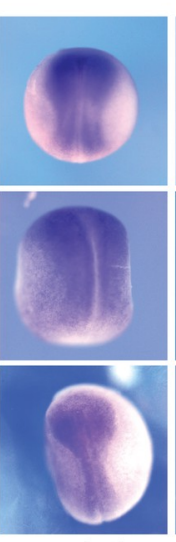

neurulation stages $25-26$
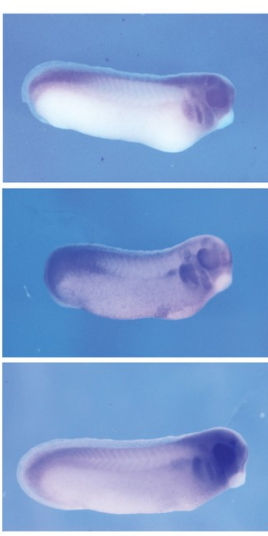

tailbud stage 32

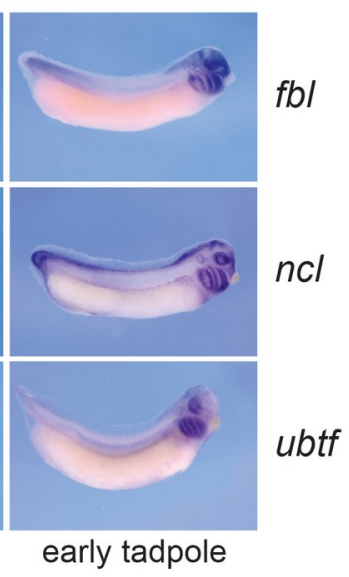

neurulation
B

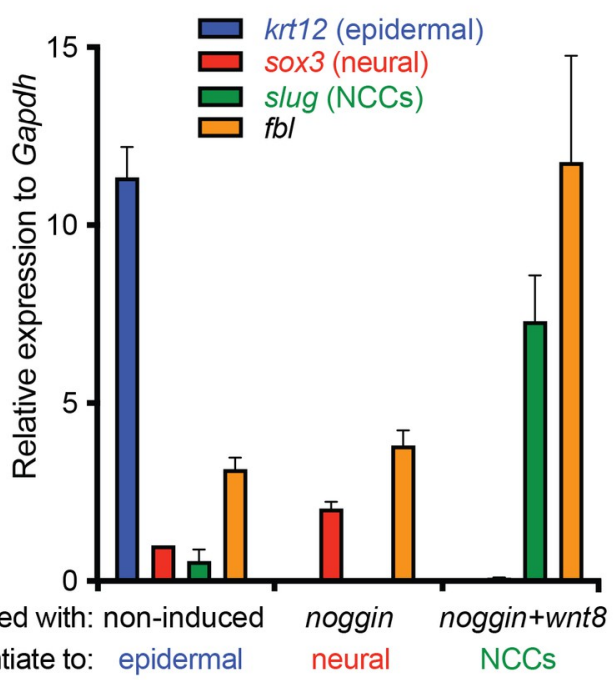

injected with: non-induced noggin

differentiate to: epidermal

neural
NCCS

stage 18:

neural plate.

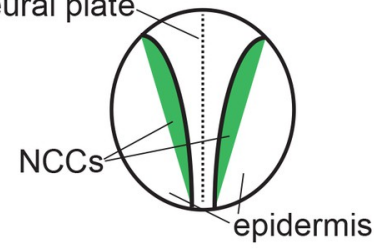

stages 25-32:

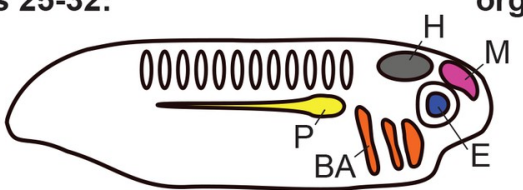

C
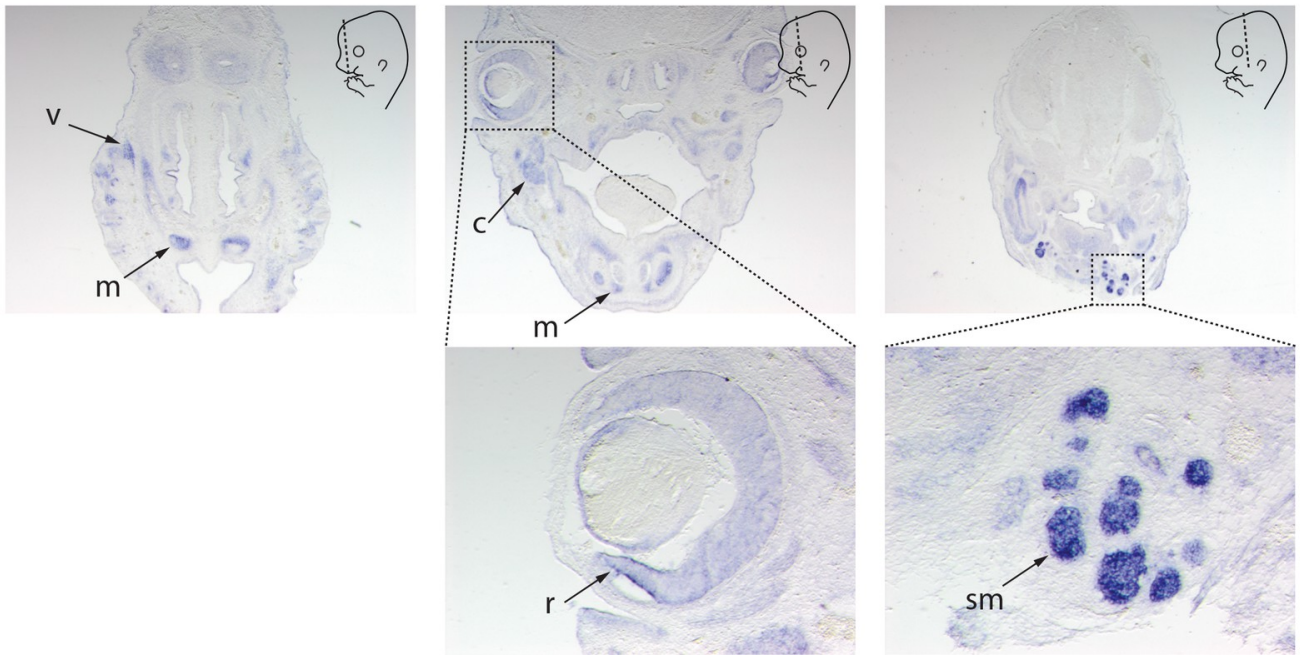

Fig 1. Spatiotemporal expression of $f b l, n c l$, and $u b t f$ in Xenopus laevis during development. $\mathbf{A}, f b l, n c l$, and $u b t f$ mRNAs were detected in X. laevis during development by whole mount in situ hybridization (WISH). The stages and, where relevant, the animal and vegetal poles are indicated. The schematics highlight the labeled structures at stage 18 (neurulation) and at stages 25-32 (organogenesis). NCCs, neural crest cells; H, hindbrain; M, midbrain; E, eyes; P, pronephros; BA, branchial arches. B, fbl expression was analyzed by RT-qPCR in animal 
cap explants injected either with noggin (to induce differentiation to neural tissue detected with sox3) or with noggin and wnt8 (to stimulate differentiation to neural crest tissue detected with slug). Non-induced (non-injected) cap explants naturally differentiate to epiderm detected with $k r t 12$. As a control, expression of the inspected genes was normalized to $g a p d h$. $f b l$ was most abundantly expressed in neural crest tissue. C, Spatial distribution of $f b l$ mRNAs established by WISH in E14.5 mouse embryos. The schematics indicate the orientation of the section plane (dashed line). v, vibrissae; $m$, mesenchyma; c, primordium of mandibular cartilage; $r$, retina; sm, submandibular glands.

https://doi.org/10.1371/journal.pgen.1010012.g001

pigmented epithelium (RPE) was measured and found to be reduced by 10-25\% (S2 Fig and S1 Data). No such eye or craniofacial cytoskeleton alterations were observed in embryos injected with a non-targeting control MO (Ctrl). The morphology of the craniofacial cartilage was further investigated by alcian blue staining (Fig 2C). This further revealed in the morphants a striking reduction, or near-complete loss in the case of Fbl depletion, of the branchial arches (Fig 2C, arrowheads). The amount of residual endogenous Fbl after MO treatment was established by western blotting as $\sim 31 \%$ ( one-third) of the normal level (Fig 2D, lane 2).

To prove that the observed developmental defects were caused by Fbl depletion, we performed rescue assays. When the $f b l \mathrm{MO}$ was co-injected with an in vitro-synthesized mRNA encoding Flag-tagged wild-type Fbl (Flag-Fbl-wt), the eye size, the area occupied by the retinal pigmented epithelium, the presence of the branchial arches, and the symmetry of craniofacial skeleton were all largely restored (Fig 2B, 2E and S2 Fig and S1 Data). Note that the rescue transcript was made resistant to MO interference by modifying its 5' untranslated region (see Materials and Methods). Efficient expression of Flag-tagged-fibrillarin was confirmed by western blotting (Fig 2D, lane 3). In rescued Fbl morphants, about half-sized normal branchial arches were formed (Fig $2 \mathrm{E}$, green arrowheads). This highly distinctive phenotype was never observed in embryos treated only with $f b l$ MO. Such partial morphological rescue is compatible with the amount of Fbl detected in rescued morphants: counting both the endogenous residual protein and the Flag-tagged construct, this amounted to $\sim 49 \%$ of the normal level (Fig 2D).

At this stage, it was interesting to probe if the methyltransferase function of $\mathrm{Fbl}$ is important for morphological rescue. Considering that the residual amount of endogenous $\mathrm{Fbl}$ in the morphants is about one-third of the normal amount, testing this possibility was always going to be challenging. Nonetheless, as an initial approach, we expressed the $f b l$-D238A mutation instead of the wild-type allele in our rescue assay. In the atomic resolution structure of the Archaeoglobus fulgidus fibrillarin-Nop5 complex bound to its cofactor and methyl donor $S$-adenosyl- ${ }_{\mathrm{L}}$ methionine (AdoMet), it has been shown that Asp-133 (equivalent to Xenopus laevis D238) is situated within $3.5 \AA$ of the thiomethyl carbon of the bound AdoMet. This implies that it plays a role as a catalytic residue [48] (see S3 Fig for the position of the residue in the catalytic pocket of the human protein). When this residue was mutated into an alanine, the methylation activity of fibrillarin was indeed totally abolished in an in vitro methylation assay [49]. Interestingly, expression of Flag-Fbl-D238A in morphants rescued the phenotypes (eye size, craniofacial skeleton symmetry, and branchial arches) to the same extent as did Flag-Fbl-wt. In particular, half-sized branchial arches were formed (Fig $2 \mathrm{~B}$ and $2 \mathrm{E}$ ). This indicates that providing maturing embryos with an additional supply of Fbl (in this case $~ 10 \%$, see Fig 2D, lane 4), even if it is not catalytically active, was sufficient to restore development partially.

We conclude that all three tested ribosome biogenesis factors, Fbl, Ncl and Ubtf, are important for eye and craniofacial skeleton development in Xenopus.

\section{Fibrillarin depletion reduces expression of neural border and neural crest markers}

To understand the origin of the eye and craniofacial skeleton defects observed upon Fbl depletion, we analyzed the distribution of a series of prototypic developmental markers. Embryos 
A

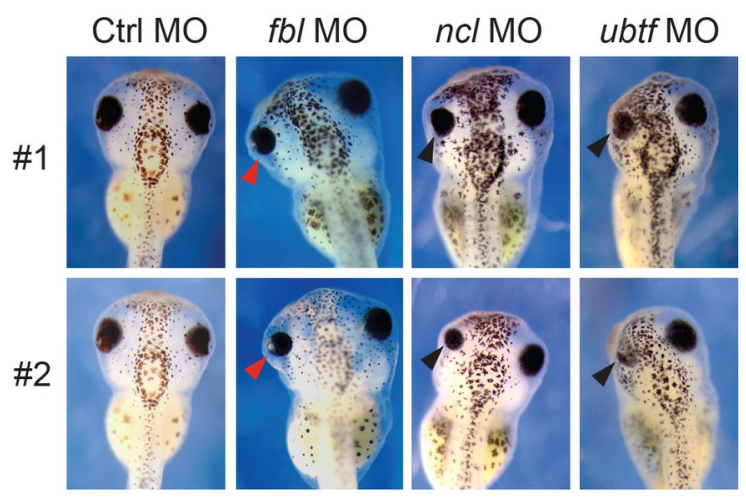

B

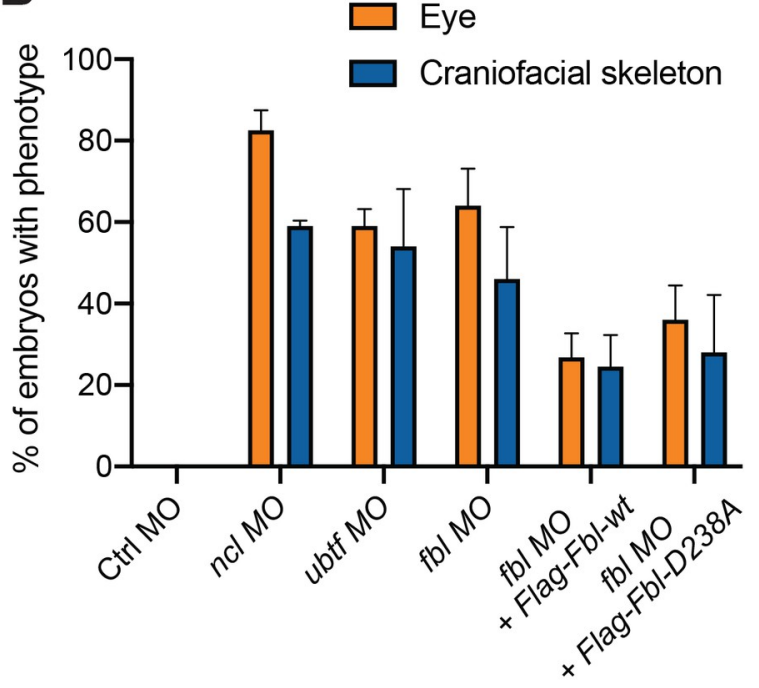

C

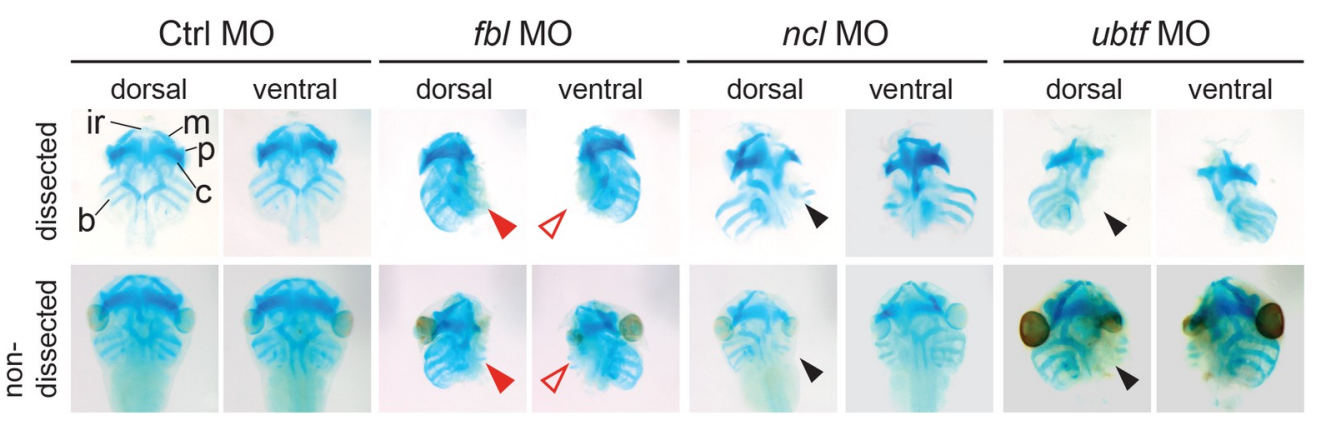

D

E
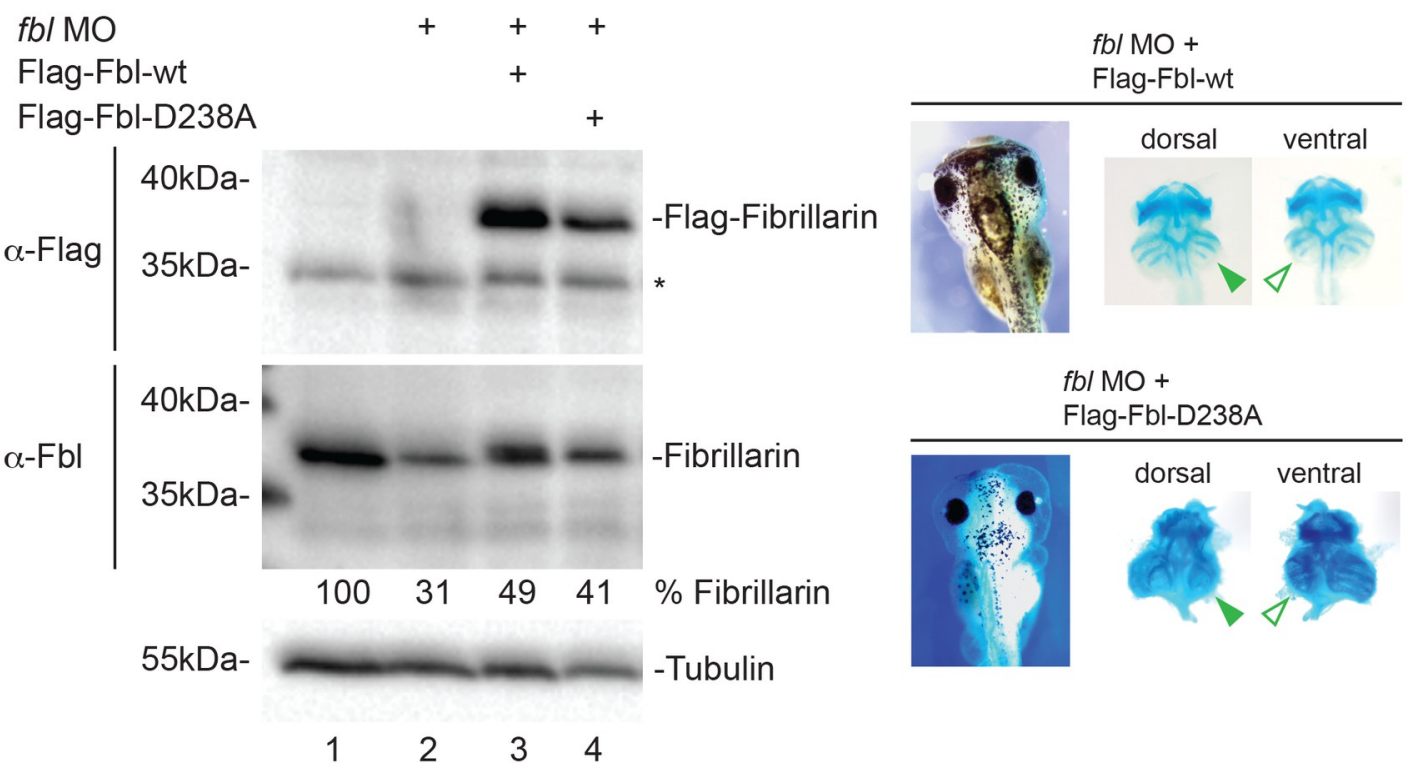

Fig 2. Morphological defects during Xenopus laevis development upon Fbl, Ncl, or Ubtf depletion. A, Embryos injected unilaterally at the two-cell stage with the indicated morpholino (MO) were inspected under the microscope at stage 42 . As a control, a non-targeting morpholino (Ctrl) was used. Two embryos are presented (\#1 and \#2), with the left side injected. With the Ctrl morpholino and those targeting $u b t f$, and $n c l$ expression, two independent experiments were performed, the total number of embryos tested being 100, 94 and 77 , 
respectively. With the morpholino targeting $f b l$ expression, a total of 154 embryos were analyzed in three independent experiments. B, Quantification of the data presented in panels A and E. Percentage of embryos displaying reduced eye size, or craniofacial skeleton defects (head asymmetry). C, Cartilage staining with alcian blue revealed a defect in all the branchial cartilages, namely: ir, infrarostral; $\mathrm{m}$, meckel; p, palatoquadrate; c, ceratohyal; b, other branchial cartilages. Non-dissected and dissected embryos are presented for comparison. Arrowheads highlight striking reduction, or near-complete loss of the branchial arches. D, Western blot analysis of Fbl-depleted and rescued morphants. Total protein was extracted from individual neurula embryos; one embryo equivalent was loaded per lane. The blots were probed with an anti-Flag antibody (top panel) or an anti-Fbl antibody (bottom panel). The anti-Flag antibody detects only the rescue constructs (Flag-Fbl-wt or Flag-Fbl-D238A). The asterisk $\left({ }^{*}\right)$ denotes detection of an aspecific band. The anti-Fbl antibody detects both the endogenous protein and the rescue constructs. The percentage of $\mathrm{Fbl} \mathrm{expressed} \mathrm{with} \mathrm{respect} \mathrm{to} \mathrm{the} \mathrm{uninjected} \mathrm{control} \mathrm{is} \mathrm{indicated.} \mathrm{The}$ western blots displayed are representative of four independent experiments. As loading control, the blots were probed for alpha-tubulin. $\mathbf{E}$, Both the wild-type Flag-Fbl and the catalytically deficient Flag-Fbl-D238A restored formation of branchial arches to about half the normal size. Representative example of an embryo co-injected with $f b l \mathrm{MO}$ and either a MO-resistant wild-type rescue construct (top) or the same construct harboring the D238A mutation (bottom). A total of 77 and 65 embryos were analyzed in two independent experiments with the Flag-Fbl-wt and Flag-Fbl-D238A construct, respectively.

https://doi.org/10.1371/journal.pgen.1010012.g002

were injected unilaterally at the two-cell stage with $20 \mathrm{ng} \mathrm{MO}$ targeting $f b l$ transcripts. The injected embryos were fixed at the neurula stage (stage 15-18) or at organogenesis (early tadpole stage 32) and analyzed by WISH to reveal expression of selected markers (Fig 3A and 3B).

Expression of the neural plate border genes $m s x 1$ and hairy 2 and of the neural crest specifier gene twist was reduced on the injected side of the embryos (Fig 3A). This was observed at both the neurula and early tadpole stages for hairy 2 and $t w i s t$, and at the neurula stage for $m s x 1$ (Fig 3A). In contrast, expression of krt12 (an epidermal marker), sox2 (a pan-neural plate marker), pax2 (forebrain), pax6 (eye), and also pax8 (otic placodes and pronephros) and prdm12 (cranial placodes) all appeared unaffected at the neurula stage. Of these, only pax6 was downregulated in later-stage embryos (stage 32).

Importantly, the downregulation of twist, observed in neurula embryos, and that of pax6, observed in the eye in tadpole embryos, were both rescued by co-injection of the MO-resistant wild-type $f b l$ construct (Fig 3B). Co-injection of the $f b l-D 238 \mathrm{~A}$ construct also restored proper expression of twist and pax6 at these stages (Fig 3B).

In order to provide initial mechanistic insights into the cellular basis of the phenotypes observed in frog upon fibrillarin knockdown, we assessed apoptotic DNA fragmentation by performing terminal deoxynucleotidyl transferase dUTP nick end labeling (TUNEL) assays (Fig $3 \mathrm{C}$ ). The $f b l \mathrm{MO}$ was injected unilaterally at the two-cell stage and TUNEL was applied to whole-mount embryos once they reached the neurula stage. TUNEL revealed important levels of DNA fragmentation in the injected sides of the embryos, particularly obvious at the neural plate, where $f b l$ is normally highly expressed. This indicates massive apoptosis. Interestingly, the increased TUNEL staining was suppressed when the $f b l \mathrm{MO}$ was co-injected with an antip53 MO. This is consistent with the role of p53 in promoting apoptosis. As a control, embryos were unilaterally injected with a transcript expressing DMRT5, whose overexpression has been shown to promote apoptosis under these conditions [50].

Thus, Fbl depletion affects neural crest survival as a result of massive p53-dependent apoptosis. Altogether, our results also indicated that the head and eye defects of Fbl morphants are a likely consequence of altered cranial neural crest cell development, as these cells start to migrate into and become patterned within the facial primordia at the neurula stage, coinciding with the observed increased apoptosis.

\section{Fibrillarin is required for efficient pre-rRNA processing in $X$. laevis}

The involvement of fibrillarin in pre-rRNA processing has been established in a variety of eukaryotic models, ranging from budding yeast to human cells. In these models, the protein was shown to be important for the initial pre-rRNA cleavages leading to synthesis of $18 \mathrm{~S}$ rRNA, the RNA component of the small ribosomal subunit [45-47]. These cleavages largely 
A
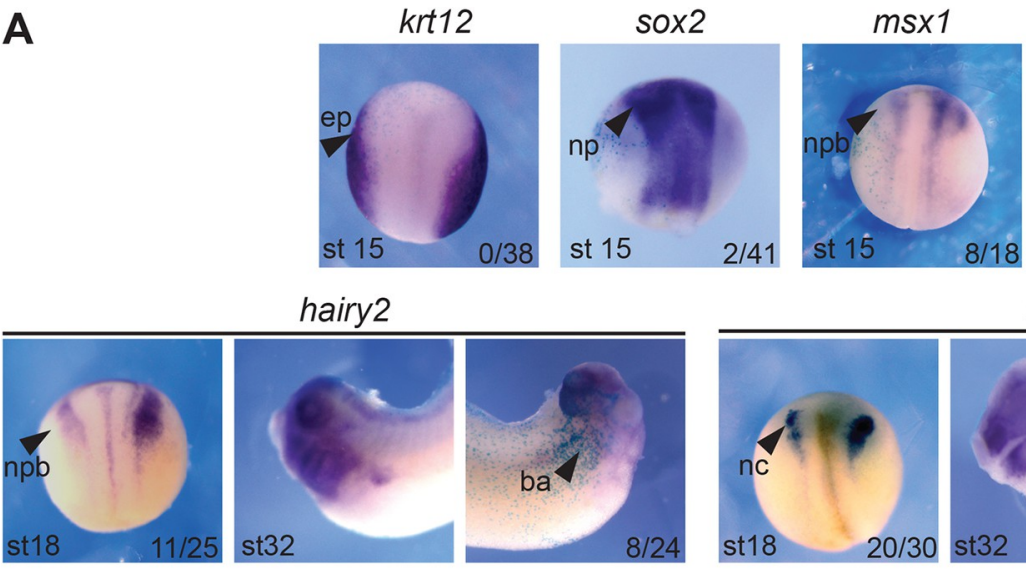

hairy2
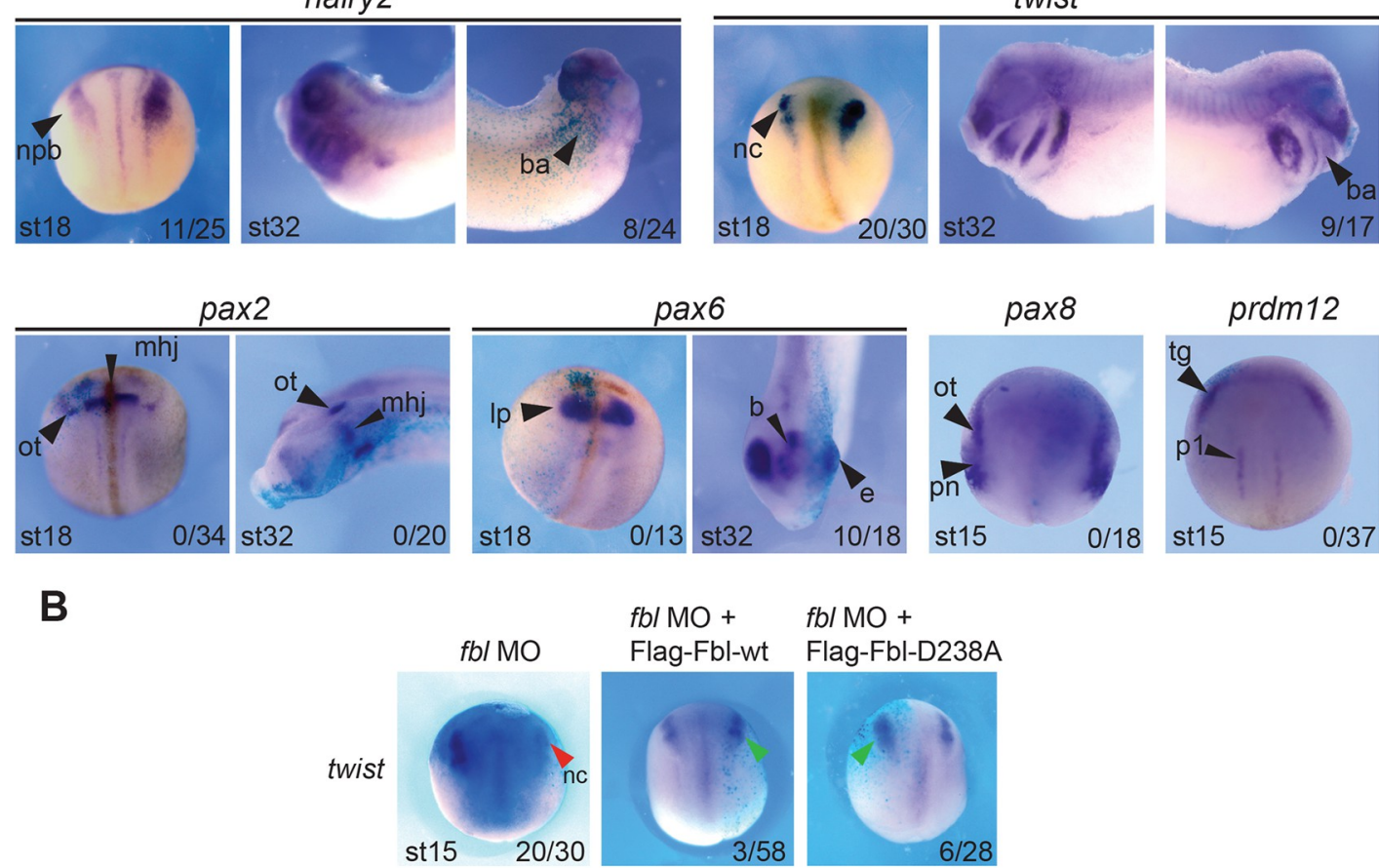

pax6
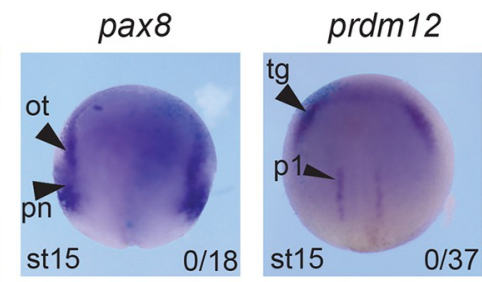

B
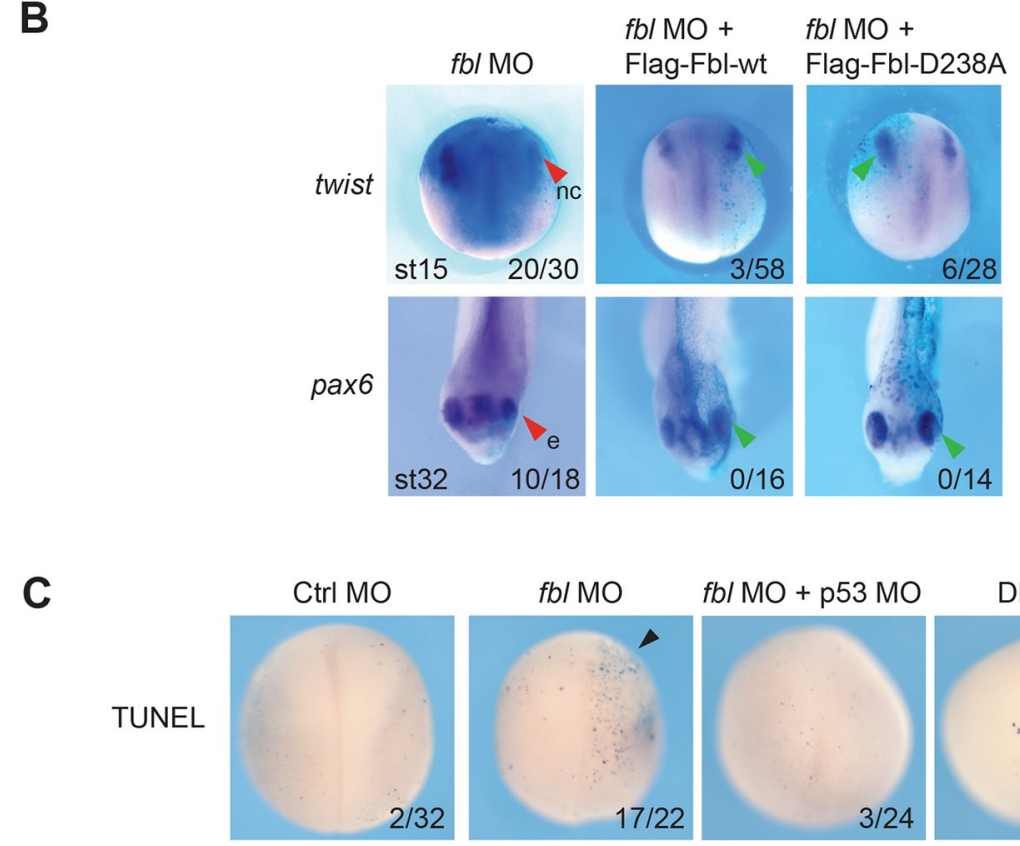

DMRT5

Fig 3. The distribution of developmental markers is affected upon Fbl depletion in Xenopus laevis. A, Expression patterns of a series of representative developmental markers established by WISH in embryos asymmetrically depleted of Fbl. The embryos were observed at the neurula stage (stages 15-18) or at organogenesis (early tadpole, stage 32). ep, epidermis; np, neural plate; npb, neural plate border; ba, branchial arches; nc, neural crest; ot, otic placode; mhj, midbrain-hindbrain junction; Ip, lens placode; b, brain; e, eye; pn, pronephros; tg, trigeminal placode; $\mathrm{p}$, V1 interneuron progenitors of the spinal cord. The injected side of each embryo was identified by co-injecting lacZ mRNA. B, The altered distribution of twist and pax6 transcripts, observed upon Fbl depletion, was restored by co-injecting embryos with $\mathrm{MO}$ and an mRNAs encoding the wildtype protein (Flag-Fbl-wt) or the D238A (Flag-Fbl-D238A) mutant. Legend as in panel A. The injected side of the embryos was identified by co-injecting lac $Z$ mRNA. In panels $A$ and $B$, the penetrance of the phenotype (number of embryos with reduced expression of the marker $v s$. the total number of observed embryos) is indicated at the bottom right of each panel. C, Detection of apoptotic DNA fragmentation by TUNEL. Embryos were unilaterally injected with $f b l \mathrm{MO}$ alone or together with p53 MO. As controls, embryos were injected with a Ctrl MO or with a transcript encoding DMRT5, known to promote apoptosis. The number of embryos with increased apoptosis $v s$. the total number of observed embryos is indicated. 
rely on the box C/D snoRNA U3, conserved in Xenopus and shown in this organism to be important for these processing steps [51,52].

To test whether Fbl is involved in ribosome biogenesis in Xenopus, total RNA extracted from MO-treated embryos was analyzed by northern blotting at the early tadpole stage (S4 Fig). Probing of the membrane with specific radioactively-labeled oligonucleotides revealed several phenotypes upon Fbl depletion: 1) accumulation of the primary ribosomal transcript (40S), 2) reduction of $20 \mathrm{~S}$ pre-rRNA, a precursor of the $18 \mathrm{~S}$ rRNA, and 3) reduced production of mature $18 \mathrm{~S}$ rRNA (S4 Fig). These phenotypes are consistent with inhibition of early prerRNA cleavages and confirm an evolutionarily conserved role of Fbl in small ribosomal subunit biogenesis in Xenopus.

During the early steps of ribosomal subunit assembly, fibrillarin binds to nascent pre-rRNA transcripts. This early binding step must occur for subunit biogenesis to proceed faithfully (RNA processing) and, importantly, it is independent of its catalytic activity in methylation ([53]). In agreement with our catalytic mutant rescue analysis (Fig 2E), we conclude it is the involvement in pre-rRNA processing of fibrillarin which is responsible for the early developmental defects reported. Furthermore, ribosome biogenesis dysfunctions, including pre-rRNA processing inhibitions, are well-established as triggering nucleolar surveillance leading to p53 stabilization (discussed in [54]). The p53-dependent apoptosis observed by TUNEL (Fig 3C) is thus explainable by the involvement of $\mathrm{Fbl}$ in pre-rRNA processing.

\section{Systematic mapping of 2'-O-methylated ribose in $X$. laevis ribosomal RNAs}

Fibrillarin modifies pre-rRNAs, aided by box C/D snoRNAs acting as antisense guides [55]. In pioneering work, Prof. Maden in Liverpool, U.K. mapped the sites of 2'-O methylation on X. laevis ribosomal RNAs, using RNase finger-printing by 2-D thin-layer chromatography [5658]. Although quite laborious, relying on the use of heavily radioactively-labeled RNAs, and offering only limited resolution, this approach enabled this investigator, nonetheless, to map 89 of the 98 2'-O methylation sites predicted at the time [56-58]. It remained unclear, however, where on the ribosome the nine remaining sites might lie, whether additional sites exist (the sensitivity of the technique was good but some sites might have escaped detection), and most importantly, what proportion of ribosomes in a population of particles is modified at each sites.

At this stage, although we had established it is the role of fibrillarin in pre-rRNA processing which is important for early development, we nonetheless felt it was interesting to reassess systematically in a quantitative fashion sites of ribosomal RNA 2'-O methylation. Such analysis might reveal undocumented variation at specific positions, possibly at particular stages of development.

To investigate systematically the 2'-O methylation of rRNAs in X. laevis, we applied RiboMethSeq, a deep sequencing-based mapping technique relying on differential sensitivity of 2'O methylated nucleotides to mild alkaline hydrolysis [59,60]. RiboMethSeq does not only afford mapping rRNA sugar methylation with single nucleotide resolution, it is also particularly powerful for quantitative assessment [14-16]. Specifically, we wanted to establish a comprehensive repertoire of all sites of 2'-O methylation in X. laevis $\mathrm{rRNAs}$ (possibly identifying novel sites), to evaluate quantitatively the level of modification at each modified position (possibly identifying sites of hypomodification), and to test the possibility that individual sites might be differentially modified during development.

We selected for analysis six stages across early development: segmentation (stage 2), gastrulation (stage 10), neurulation (stage 16), early organogenesis (tailbud, stage 23 and tadpole, stage 32), and late organogenesis (stage 45). As RiboMethSeq requires only minute amounts of 


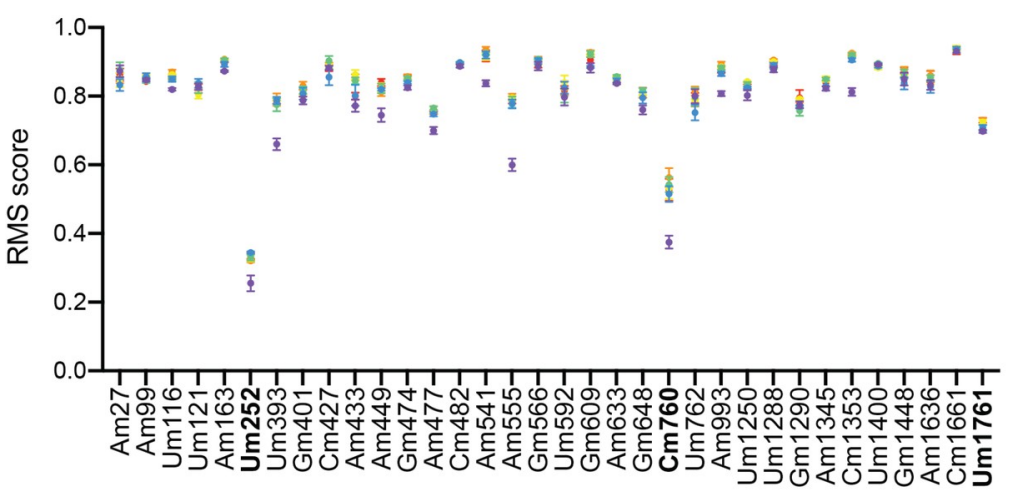

$18 \mathrm{~S}$ rRNA
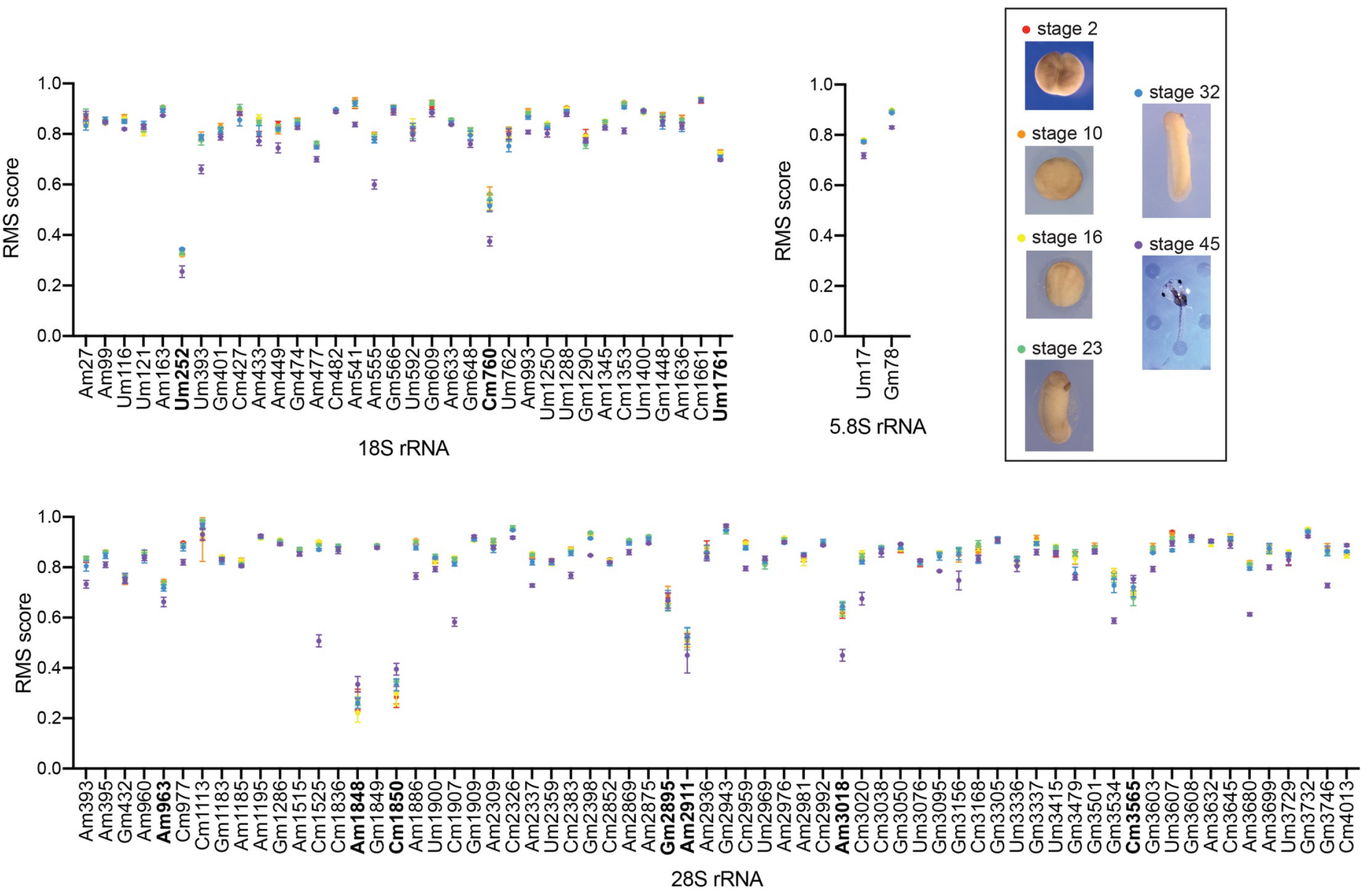

Fig 4. RiboMethSeq analysis of Xenopus 18S, 5.8S, and $28 S$ rRNAs. The methylation status of rRNAs was investigated systematically by RiboMethSeq at six developmental stages (stages 2, 10, 16, 23, 32, and 45). At each stage, RNA extracted from four individual embryos was sequenced independently. X axis, modified nucleotides. Y axis, methylation scores (see S1 and S2 Tables for values). In bold, positions hypomodified at all stages (using a cut-off of 0.75). Inset, representative pictures of embryos at each analyzed stage are displayed.

https://doi.org/10.1371/journal.pgen.1010012.g004

total RNA [60], for each stage analyzed we used RNA extracted from a single embryo. For consistency, four embryos were analyzed individually at each selected stage (Figs $\underline{4}$ and $\underline{S}$ ). Briefly, total RNA was extracted, fragmented by means of mild alkaline treatment, and converted to a deep sequencing library. Libraries were loaded onto a HiSeq1000 sequencer. Following alkaline fragmentation, RNA fragments starting or ending with a 2'-O methylated residue are strongly underrepresented because the phosphodiester bond adjacent to the modification is resistant to cleavage. Mapping of the millions of reads (15 to 25 million) onto the reference rRNA sequences made it possible to infer which position was methylated and the extent of modification at each site (methylation score) [60]. Note that for $28 \mathrm{~S}$ rRNA, we observed several sequence differences between the reference $X$. laevis sequence in the database and the sequence of our animals. We therefore deposited a revised 28S sequence at NCBI under ref. MT122800. For the other rRNAs, the reference sequences and ours were the same.

The results for 18S, 5.8S, and $28 \mathrm{~S}$ rRNAs are displayed in Fig 4 . The complete dataset is available in S1 and S2 Tables. Cluster analysis revealed, for each of the six developmental stages analyzed, clear clustering of the methylation scores obtained for the four individual embryos (S5 Fig), revealing that our dataset is extremely robust. 


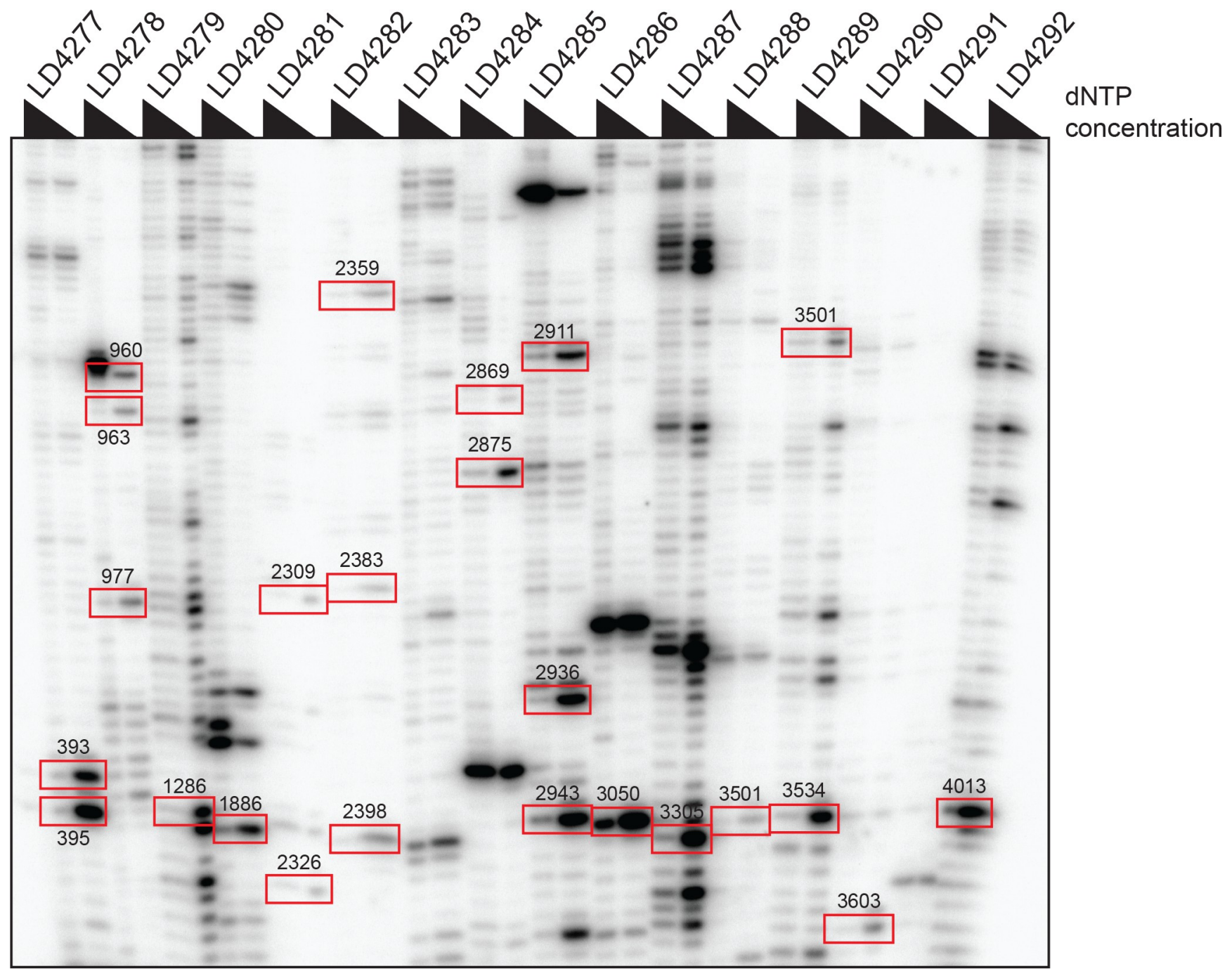

Fig 5. Orthogonal validation by low dNTP primer extension analysis of newly identified 2'-O methylated positions on Xenopus 28S rRNA. Low dNTP primer extension has proved efficient for specifically detecting 2'-O methylated nucleotides [61]. Total RNA extracted from stage $23 \mathrm{X}$. laevis embryos was analyzed by primer extension using regular or low dNTP conditions with sixteen oligonucleotides (LD4277 to LD4292) designed to survey 23 positions on the $28 \mathrm{~S}$ rRNA. A position was confirmed when the signal obtained with low-concentration dNTP was higher than that observed with the regular concentration. The analysis confirmed all 23 positions inspected (highlighted by red squares). Note that position 3501 was confirmed twice, once with oligonucleotide LD4288 and once with LD4289.

https://doi.org/10.1371/journal.pgen.1010012.g005

Several conclusions were drawn from our RiboMethSeq analysis:

Firstly, the analysis confirmed all 89 of the previously mapped 2'-O methylated positions.

Secondly, we identified 15 novel putative 2'-O methylated residues: one on $18 \mathrm{~S}$ and fourteen on $28 \mathrm{~S}$ rRNA (Fig 4 and S1 and S2 Tables). We confirmed ten of these new positions, as well as thirteen of the previously mapped ones, by use of an orthogonal mapping technique, namely: low dNTP primer extension detection [61] (Fig 5). The orthogonally-confirmed novel positions are the 28S nucleotides Am393, Am395, Am960, Gm1286, Am1886, Gm2398, Gm2943, Gm3050, Gm3305, and Gm3501 (Fig 5 and S1 and S2 Tables). The previously mapped positions which we confirmed orthogonally are the 28S residues Am963, Cm977, Am2309, Cm2326, Um2359, Cm2383, Am2869, Am2875, Am2911, Am2936, Gm3534, Gm3603, and Cm4013.

A bioinformatic search of the X. laevis transcriptome, assisted by the Snoscan software, allowed us to assign box C/D snoRNA guides to all the 2'-O methylated positions identified by 
RiboMethSeq (S3 Table). We identified a total of 49 novel X. laevis box C/D snoRNA candidates (S3 Table).

Thirdly, using as cut-off a methylation score of 0.75 , we observed that numerous positions are not fully modified (Fig 4 and S1 and S2 Tables). This indicates that in X. laevis as in other investigated experimental models such as budding yeast, zebrafish, mouse, and human, cells produce heterogeneous populations of ribosomes with differentially modified ribosomes that might display specific translational properties (see Discussion).

Lastly, close inspection of the methylation scores at individual positions through development revealed that specific nucleotides are differentially modified as the embryos develop (Fig 4 and S1 and S2 Tables), with a trend towards reduced methylation levels at specific positions. At stage 45 (highlighted in purple in Fig 4), this was notably the case at one position in 5.8S, three positions in $18 \mathrm{~S}$, and eight in $28 \mathrm{~S}$.

Of particular interest is the $18 \mathrm{~S}$ rRNA Um 252 modification, a newly identified position in $X$. laevis which is not modified in human cells and which is hypomodified at all the stages inspected. Other positions of interest on $18 \mathrm{~S}$ rRNA are: (1) Cm760, which is hypomethylated throughout development (interestingly, the corresponding position is also hypomethylated in cultured human cells [15]); (2) Um1761, which is hypomodified throughout development whereas its human counterpart is fully methylated [15]; (3) three additional positions which are hypomethylated only at stage 45 (Um393, Am477, Am555). The equivalent human positions are also hypomodified, at least in some cell lines investigated [14,15,17]. On the $28 \mathrm{~S}$ rRNA, we noted that: (1) seven positions are hypomodified at all developmental stages analyzed (Am963, Am1848, Cm1850, Gm2895, Am2911, Am3018, Cm3565), the equivalent human positions of five of these also being hypomodified [14,15]; (2) eight positions which are hypomethylated only at stage 45 (Am393, Cm1525, Cm1907, Am2337, Cm3020, Gm3534, Am3680, Gm3746). In the human cell lines tested, the equivalent positions of three of these (Cm1881, Cm3869, and Am4571) are also hypomethylated [15,17].

\section{Discussion}

\section{Neural crest cell survival requires efficient ribosome biogenesis in Xenopus}

With the emergence of ribosomopathies, a novel class of human syndromes caused by ribosome biogenesis dysfunction, investigators have become aware of the importance of understanding how tissue-specific diseases can involve a process as global as ribosome biogenesis [26]. In this work, we have studied the involvement of the box C/D snoRNA-associated methyltransferase fibrillarin in the early development of $X$. laevis. We have established the spatiotemporal expression of fibrillarin, revealing it to be abundantly expressed in neural crest cells and cells derived from them (Fig 1). This is also true of two other factors involved in distinct ribosome biogenesis steps, $\mathrm{Ncl}$ and Ubtf. We have shown that morpholino-mediated depletion of Fbl strongly impacts the survival of NCCs, with dramatic repercussions on eye and craniofacial skeleton formation leading to head dysmorphism caused by severe branchial arches alterations (Fig 2). We have corroborated these developmental defects by demonstrating altered expression of several markers of NCC formation (hairy2, msx1, and twist) and by detecting increased apoptosis at the neural plate (Fig 3).

\section{Why are ribosome biogenesis factors more abundantly expressed in neural crest cells and derivatives?}

A straightforward explanation might be that specific parts of the embryo rely on more abundant protein production to develop, and thus on more active ribosome biogenesis. Another 
possibility is that specific parts of the embryo have differential requirements for specific ribosome biogenesis factors, possibly to assemble distinct ribosomes. Although this second hypothesis is quite appealing, we do not particularly favor it at this stage, because the spatiotemporal expression profiles of the three ribosome biogenesis factors inspected in this work largely overlap and because these are core factors playing evolutionarily conserved essential roles in ribosome biogenesis. This view is strengthened by the observation that additional ribosome assembly factors, and also several ribosomal proteins, have been shown to share, during early development, expression patterns largely similar to that observed here for $\mathrm{Fbl}, \mathrm{Ncl}$, and Ubtf [36-39,62].

During development in the mouse, $\mathrm{Fbl}$ is essential at a very early stage, prior to embryo implantation [44]. The mechanisms involved and the precise spatiotemporal expression of $\mathrm{Fbl}$ in mouse embryos remained undefined. Here we have clarified the pattern of expression of $\mathrm{Fbl}$ at high resolution in E14.5 mouse embryos, finding it to be consistent with our observations on X. laevis (Fig 1C). In zebrafish, fibrillarin is preferentially expressed in the proliferating cells of the optic tectum, in the retina, and in the dorsal midbrain, and it is important for the maturation of these regions [47]. These observations on a fish are perfectly consistent with our own findings in X. laevis (Figs 1 and 3). The function of fibrillarin in pre-rRNA processing is conserved in yeast [45], human cells [46], and zebrafish [47], and now we show it to be conserved also in X. laevis (S4 Fig).

There are currently two models for explaining how a global process such as ribosome biogenesis can lead to tissue-specific disorders: the 'specialized ribosomes' model and the 'ribosome concentration' model. The specialized ribosome model posits that compositionally distinct ribosomes, such as those harboring more or less methylated residues, have differential translational capacities [63]. The ribosome concentration model proposes, rather, that the amount of functional ribosomes is what dictates which mRNAs are preferentially translated. In this case, the rationale is that naturally 'less favored' mRNAs will be even less translated when the intracellular ribosome concentration drops [64]. Both of these models have qualities and both mechanisms are likely to coexist in cells, each contributing to some extent to regulating the translational capacity. Because of the pre-rRNA processing defects observed and the phenotypic rescue with a catalytically defective allele, in the case of the Fbl-mediated developmental defects reported here, our data clearly favors the ribosome concentration model.

The majority of bones, cartilages, and connective tissues comprising the head and face are derived from neural crest cells. Craniofacial skeleton alterations are conspicuous in developing animals where ribosome biogenesis is inhibited. Typically, they are observed in fish and mouse embryos with phenotypes reminiscent of Treacher-Collins syndrome, a neurocristopathy caused by mutations in factors important for rRNA synthesis $[42,65,66]$. They are also observed upon fibrillarin depletion in Xenopus, as reported here, and in both Xenopus and fish models upon depletion of several other factors intervening at different steps of ribosome biogenesis [33,38]. Altogether, this strongly supports the notion that NCC survival is particularly sensitive to the capacity of cells to synthetize ribosomes.

\section{Ribosomal RNAs are distinctly hypomethylated at late stages of Xenopus development}

On the basis of our RiboMethSeq data, we report 103 methylation sites on X. laevis rRNA: 34 on 18S, 2 on 5.8S, and 67 on $28 \mathrm{~S}$ (see S1 and S2 Tables). By comparison, human rRNAs harbor 112 positions: 42 on 18S, 2 on $5.8 \mathrm{~S}$, and 68 on $28 \mathrm{~S}$, most sites being conserved from frog to human (S1 Table). On $18 \mathrm{~S}$ rRNA, X. laevis carries the Um252 modification, which is not conserved in human cells and which is hypomodified at all stages inspected. Human $18 \mathrm{~S}$ rRNA 
contains nine modifications which are not conserved in X. laevis, namely Am159, Um172, Cm174, Um354, Cm621, Gm867, Cm1272, Gm1447, and Um1668. Among them, most are hypomodified in the human cell lines tested (Cm174, Um354, Cm621, Gm867, Cm1272, Gm1447 and Um1668). On 28S rRNA, X. laevis harbors four modifications which are not conserved in human cells: $\mathrm{Gm} 432, \mathrm{Cm} 1113, \mathrm{Gm} 3534$ and $\mathrm{Cm} 4013$. Only Cm3534 is hypomethylated at later developmental stages (32 and 45). Human $28 \mathrm{~S}$ rRNA contains five modifications that are absent in X. laevis: Gm1316, Um1773, Cm2824, Gm3627, and Gm4618. Again, most of these are hypomodified (Gm1316, Um1773, Cm2824, Gm4618).

Of the 103 positions mapped here in X. laevis, fifteen have not been detected previously [56-58]. Importantly, using of a low dNTP primer extension assay, we have orthogonally validated 23 positions, including ten of the newly identified ones (Fig 5). Furthermore, upon searching the $X$. laevis transcriptome, we have identified box C/D snoRNA candidates for all the identified positions ( $\mathrm{S} 3 \mathrm{Table}$ ). We also note that one position identified historically [56,57], Um2326, was not confirmed by our RMS or our low dNTP primer extension analysis. This position is also absent in X. tropicalis. We conclude that this position was originally misassigned.

We have profiled rRNA sugar methylation in individual embryos at six developmental stages, concluding that the vast majority $(87.6 \%)$ of positions are robustly installed, while a minority (12.4\%) are hypomethylated (cut-off score: 0.75 ). In principle, fractional ribosomal RNA 2'-O methylation implies that cells produce a heterogeneous population of ribosomes. Whether these differentially modified ribosomes are all actively involved in translation remains to be established. Ribosomal RNA hypomethylation has been observed in cancer cells [14-17], and during development in fish [18] and mouse [19]. We now report it in Xenopus. The exact significance of substoichiometric rRNA methylation remains largely unknown.

We have also observed that the degree of hypomethylation increases substantially at later stages of development. This is particularly striking at stage 45 , at three positions on $18 \mathrm{~S}$, one on 5.8S, and eight on 28S. Whether and how reduced methylation at these sites might affect translation, and possibly late stage of development, deserves future investigation.

The mechanisms of hypomethylation are not yet known. In particular, it remains unclear whether sites of hypomethylation are hardcoded or whether they simply reflect stochastic elements inherent in ribosomal subunit biogenesis. What we have learned so far with cancer cells is that not all sites vary to the same extent [15]. In particular, sites located at the periphery of a ribosomal subunit are more prone to variation than those located at the core of the ribosome, where the functionally important sites lie. Thus, only a limited number of positions are subjected to partial modification.

We have also learned that upon controlled and progressive depletion of fibrillarin in human cells, the sites which become less modified are largely those which are naturally hypomodified [15]. This clearly indicates that some sites are more easily modified than others. It also provides a simple mechanistic explanation of hypomethylation: under conditions of active cell proliferation, as in cancer cells or at specific developmental stages, the rRNA modification machinery might be 'overwhelmed' by increased ribosome production, and snoRNPs might be insufficient in number or might find it hard to access their binding sites within the complex folding 3-D structure of the maturing ribosomal subunits. In agreement with this hypothesis, profiling of rRNA 2'-O methylation in differentiated tissues, where cell proliferation is reduced as compared to cultured cells, shows most sites to be robustly methylated $[18,19]$.

In a recent work, the team of Prof. Gall used RNA-sequencing data from the giant oocyte nucleus of $X$. tropicalis to annotate snoRNAs and to compare his findings with those pertaining to other vertebrates [67]. In their analysis, the authors identified in X. tropicalis 102 2'-O methylated positions and 99 box C/D snoRNAs. They predicted novel modified positions, 
some of which were investigated in a complex orthologous system consisting of a yeast strain deleted of the endogenous snoRNA guide and provided with an exogenous frog guide expressed from a plasmid. Methylation of the predicted substrate was then verified by low dNTP primer extension [67], which is non- quantitative. While snoRNP machineries are well conserved, the use of a heterologous system might not be optimal in all cases because of species specificities. It also raises questions regarding the copy number of the complementing exogenous snoRNA, particularly in cases of weak anti-sense guide elements which might not allow modification when expressed at physiological levels. By comparison, we have conducted a comprehensive survey of rRNA 2'-O methylation directly on X. laevis rRNAs extracted from individual developing animals. For this we have used the highly quantitative RiboMethSeq technique, subsequently assigning snoRNA guides to the known and newly identified positions.

In conclusion, we have shown in X. laevis that neural crest cells are particularly sensitive to ribosome biogenesis inhibition, and that in the late stages of embryonic development rRNA hypomethylation strikingly increases at specific positions. Further study is required to determine whether this developmentally regulated rRNA hypomethylation results in differential translation of particular mRNA transcripts important for controlling late developmental transitions.

\section{Materials and methods}

\section{Ethics statement}

Animal housing and experimental protocols were approved by the CEBEA ("Comité d'éthique et du bien être animal") of the IBMM-ULB and conformed to the European guidelines on the ethical care and use of animals.

\section{Expression plasmids and morpholinos}

The $f b l, u b t f$, and $n c l$ expression vector were constructed by PCR from $X$. laevis pCMV-SPORT6-FBL (mRNAID: XM_018228388.1), pCMV-SPORT6-UBTF (mRNAID: NM_001101787.1), and pCR4-TOPO-NCL (mRNAID: NM_001088088.1), using primers described in S4 Table. The PCR products were cloned into the BamH I and Xho I sites of the pCS2 vector. To deplete Ubtf, fibrillarin, and nucleolin, we used an antisense morpholino specific to sequences directly upstream of their ATG. Morpholinos were purchased from GeneTools (Philomath, OR) (S5 Table).

Fibrillarin rescue constructs: We produced two fibrillarin rescue constructs, one encoding the wild-type allele, the other encoding the D238A mutation (see S3 Fig). mRNA was produced from each construct by in vitro transcription, it was capped, poly-adenylated, and purified on gel. The purified mRNA was injected directly into the embryos (see below). The rescue constructs were resistant to the morpholinos because the sequence upstream of the ATG was modified so as to lose its complementarity to the antisense oligo. To distinguish endogenously expressed fibrillarin from protein produced from the injected mRNAs, a Flag-tag was inserted in-frame at the amino-terminus of the rescue constructs. The wild-type construct was amplified with primers LD3036 and LD2749 and cloned into pCS2-Flag between the EcoR I and the Xho I site. The D238A catalytically deficient $f b l$ mutant was generated by site-directed mutagenesis with the help of the QuikChange II site-directed mutagenesis kit (Stratagene) and primers LD3881 and LD3882. Oligonucleotides are listed in S4 Table.

\section{Embryos, micro-injections, and $X$. laevis animal cap explant culture}

Xenopus embryos were obtained from adult frogs by hormone-induced egg-laying. Embryos were generated by in vitro fertilization [68] and staged according to [69]. For in situ analysis, 
embryos were injected in one blastomere at the $2-4$ cell stage (200 pg mRNA or $10 \mathrm{ng}$ of morpholino per blastomere) and fixed at the neurula or tailbud stages. X. laevis wnt8 (50 pg/blastomere [70]), noggin (100 pg/blastomere [71]), Flag-fbl-wt (250 pg/blastomere), and Flag-fblD238A (250 pg/blastomere) mRNAs were synthesized with the mMessage mMachine kit (Ambion). To reveal the injected side, embryos were co-injected with lacZ mRNA (50 pg/ blastomere).

In the animal cap assays, mRNAs were microinjected into the animal region of each blastomere of four-cell-stage embryos. Animal caps were dissected at the blastula stage (stage 9) and cultured in $1 \mathrm{x}$ Steinberg medium, $0.1 \%$ BSA until sibling embryos reached the neurula stage. All Xenopus embryo injections were performed on at least three batches of embryos.

The area occupied by the RPE was calculated for both eyes with Image J and the injected to non-injected side area ratio was calculated. RPE ratios were analyzed with the KolmogorovSmirnov test (an unpaired t-test, unparamatretric and specific for cumulative distribution comparisons).

Wild-type C57BL/6 mice were crossed to generate the embryos used in this study.

\section{Lineage tracing, in situ hybridization, and cartilage staining}

Xenopus embryos were fixed in MEMFA and stained with X-gal to visualize LacZ activity on the injected side before being processed for in situ hybridization. Whole mount in situ hybridization was performed on Xenopus embryos as previously described [68]. Synthetic digoxigenin-labeled antisense mRNAs probes were prepared using the T7 polymerase and pCS2-fbl, or pCS2-ubtf, or pCS2-ncl. NBT/BCIP was used as a color substrate. For in situ hybridization on mouse embryos, $20-\mu \mathrm{m}$ cryostat sections of $4 \%$ paraformaldehyde-fixed, $30 \%$ sucrose/PBSinfused tissues frozen in gelatin (7.5\% gelatin, $15 \%$ sucrose/PBS) were used. In situ hybridization experiments were performed as previously described [72]. The mouse FBL antisense probe was generated with the corresponding EST. The other antisense probes were generated with previously described templates encoding krt12, sox2, msx1, and hairy2 [73], twist [74], pax2, and pax6 [75], and prdm12 [76]. Cartilage staining of Xenopus embryos was performed on tadpole heads as described [36].

\section{TUNEL assay}

Terminal deoxynucleotidyl transferase dUTP nick end labeling (TUNEL) detects DNA breaks formed during apoptosis. Whole mount TUNEL staining of developmentally staged (neurula) control and morphant embryos was carried out as described in [77] with the following reagents: terminal deoxynucleotidyl transferase (TdT) $(15 \mathrm{U} / \mu \mathrm{l}$ and $5 \mathrm{x}$ buffer, Thermofisher Ref. 10533065), digoxigenin-11-dUTP ( $25 \mathrm{nmol} / 25 \mu$ l, Sigma Ref. 11093088910), Normal goat serum (Thermofisher Ref. 10000C), anti-dig alkaline phosphatase conjugate (Sigma Ref. 11093274910), nitro blue tetrazolium (Sigma Ref. 11383213001), and 5-bromo-4-chloro3-indolyl phosphate (Sigma Ref. 10760994001). The control DMRT5 transcript was prepared as previously described [50].

\section{Western blot analysis}

Total protein was extracted from single Xenopus embryos lysed with a mini-pestle (Vwr, Ref. 431-0094) in liquid nitrogen in a 1.5-ml Eppendorf tube and resuspended in ice-cold lysis buffer (50 mM Tris pH 7.6, $10 \mathrm{mM}$ EDTA, $150 \mathrm{mM} \mathrm{NaCl}, 1 \%$ Triton, supplemented with complete protease inhibitor, Roche Ref. 11697498001-1 pellet per $50 \mathrm{ml}$ buffer) and incubated on ice for $10 \mathrm{~min}$. The lysate was cleared by centrifugation $\left(12,000 \mathrm{rpm}, 10 \mathrm{~min}\right.$ at $\left.4^{\circ} \mathrm{C}\right)$. One embryo equivalent was loaded onto a 15\% SDS-PAGE gel. The gels were transferred to 
nitrocellulose membranes and probed in TBS/0.1\% tween /3\% BSA. Probing was as follows: anti-fibrillarin (Abcam, Ref. ab5821) incubated overnight at 1:2000 dilution, followed by antirabbit HRP (Cytiva, Ref. NA934V) used at a 1:5000 dilution for 2 hours; anti-Flag (Sigma, Ref. F3165) incubated overnight at 1:2000 dilution followed by anti-mouse HRP (Jackson ImmunoResearch, Ref. 115-035-062) used at 1:5000 for 2 hours; anti-alpha-tubulin (Sigma, Ref. T5168) incubated overnight at a 1:5000 dilution followed by anti-mouse HRP.

\section{RT-qPCR analysis}

For RT-qPCR analysis of X. laevis animal caps, total RNA was extracted using the NETS method. The RNA samples were digested with RNase-free DNase I before RT- PCR. The amount of RNA isolated was quantified by measuring the optical density with a nanodrop spectrophotometer. The RNA samples were digested with RNase-free DNAse I before RT-PCR. cDNA was synthesized with the iScript cDNA synthesis kit (Biorad). Synthesized cDNA was amplified with the qScript One-Step SYBR Green qRT-PCR Kit (Quanta bio) with suitable primers (see S4 Table). Samples were normalized to X. laevis gapdh.

\section{Pre-rRNA analysis by northern blotting}

Total RNA was extracted from MO-treated and untreated embryos with Tri reagent. Pooled stage 32 embryos were used. $5 \mu \mathrm{g}$ total RNA was analyzed by northern blotting as previously described [46]. The probes used for the hybridization are depicted in S4 Table.

\section{Systematic mapping of ribosomal RNA 2'-O methylation by RiboMethSeq}

RiboMethSeq analysis of rRNA 2'-O-methylation was performed as described in [60]. Briefly, $\sim 100 \mathrm{ng}$ of total RNA extracted from biological material was fragmented in bicarbonate buffer at $\mathrm{pH} 9.3$ at $96^{\circ} \mathrm{C}$, fragmentation time was adapted to obtain short RNA fragments of $\sim 20-30$ nt in length. Fragmented RNA was end-repaired, to insure compatibility with adapter ligation. Library preparation was done using NEBNext Small RNA kit, according to manufacturer's recommendations. Barcoded libraries were loaded to the flow-cell and sequenced on Illumina HiSeq 1000. Raw reads were trimmed and aligned to the reference. Bioinformatics analysis was performed as described [60]. The complete data set is available at the European Nucleotide Archive under accession $\mathrm{n}^{\circ}$ PRJEB42253.

\section{Site-specific detection of 2'-O methylation by low dNTP primer extension}

Primer extension was conducted as described in [78] on $5 \mu \mathrm{g}$ total RNA extracted from stage 23 embryos. The primers used are listed in $\mathrm{S} 4$ Table.

\section{SnoRNA identification}

To find snoRNAs in RNA-seq experiments, we downloaded sequencing data from lariat intronic RNAs of X. laevis [79]. The FASTQ file was downloaded from NCBI (SRR7615230, project PRJNA479418) with the SRA Toolkit package (http://ncbi.github.io/sra-tools/, version 2.1.10): fastq-dump SRR7615230-outdir/path/to/dir. FASTQ files were converted to FASTA files with seqtk software (https://github.com/lh3/seqtk, version 1.0-1) and used as our next input: seqtk seq - a SRR7615230.fq.gz>SRR7615230.fasta. We used snoScan (version 0.9.1) [80] to detect box C/D methylation guide snoRNAs in X. laevis RNA sequencing experiments. The predicted snoRNA sequence is provided in FASTA format. The snoRNA sequence consists of four leading nucleotides followed by the box $\mathrm{C}$ sequence, the sequence complementary to rRNA, the $\mathrm{D}$ box (and D' box) sequence, and two trailing nucleotides. snoScan computes a score based on 
probabilistic models of sequence features (terminal stem, box C, guide sequence, box $\mathrm{D}$, etc.). When multiple snoRNAs were predicted for one methylation site, we chose the one with the highest snoScan average bit scores and with high similarity to a corresponding known snoRNA in X. tropicalis. To validate our results, we checked if we detected the same snoRNAs as already identified by the Rfam database [81] in X. laevis (https://rfam.xfam.org/). We aligned snoScan snoRNA candidates with Rfam snoRNAs with Clustal Omega [82] (https:// www.ebi.ac.uk/Tools $/ \mathrm{msa} /$ clustalo/) and compared sequence alignment scores.

\section{Supporting information}

S1 Fig. RT-PCR analysis of $f b l, u b t f$, and $n c l$ expression during $X$. laevis development. Total RNA extracted from embryos at the indicated stages was analyzed by RT-PCR using amplicons specific to $f b l, u b t f$, or $n c l$ transcripts (see Materials and Methods). (TIF)

S2 Fig. Relative retinal pigmented epithelium (RPE) area of data presented in Fig 2A. Relative retinal pigmented epithelium (RPE) area (Kolmogorov-Smirnov test, ${ }^{* * * *}=\mathrm{p}<0.0001$, $\left.{ }^{* *}=\mathrm{p}<0.01\right)$.

S3 Fig. Conservation of fibrillarin residues important for methyltransferase function. The residue mutated in this work (D238 in Xenopus laevis) is highlighted in red in both panels. In the atomic resolution structure of Archaeoglobus fulgidus fibrillarin-Nop5 complex bound to

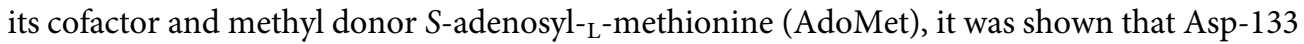
(equivalent to Xenopus laevis D238) is situated within $3.5 \AA$ of the thiomethyl carbon of the bound AdoMet, implying that it plays a role as a catalytic residue [48]. When this residue was mutated to an alanine, the methylation activity of the complex was indeed totally abolished in an in vitro methylation assay [49]. It has been suggested that Asp-133 in fibrillarin may act as a general base by deprotonating the 2'-OH group of the target RNA during catalysis. It has further been suggested that Asp-133 may also facilitate cofactor binding through favorable electrostatic interactions $[48,49]$. A, 3-D model of the catalytic pocket of human fibrillarin (based on PDB 2ipx). D238 (in red, Xenopus numbering) is directly adjacent to the AdoMet (stick representation) with the methyl group to be transferred from the cofactor to the RNA substrate represented in pink. B, Multiple alignment between fibrillarin proteins of different origins (HUMAN, Homo sapiens; XENLA; Xenopus laevis; YEAST, Saccharomyces cerevisiae; ARCFU, Archaeoglobus fulgidus; PYRFU, Pyrococcus furiosus; and METJA, Methanocaldococcus jannaschii). Residues highlighted in blue and red $(\mathrm{K} / \mathrm{D} / \mathrm{K})$ are absolutely conserved and correspond to the catalytic triad. The $\mathrm{D}$ residue in this triad is the residue mutated in this work. Bold, residues important for SAM binding. Asterisks, residues identical across all six species examined. Sequences were aligned with CLUSTAL.

S4 Fig. Xenopus fibrillarin is required for small ribosomal subunit synthesis. Total RNA extracted from stage 32 embryos injected into each cell at the 2-cell stage with $f b l \mathrm{MO}$ or with a non-targeting $\mathrm{MO}(\mathrm{Ctrl})$ was separated on denaturing agarose gel and processed for northern blotting with radioactively-labeled probes designed to detect pre-rRNA precursors. As a control, non-injected embryos were used. A, Ethidium-bromide-stained gel. Note that the mature $18 \mathrm{~S}$ and $28 \mathrm{~S}$ rRNAs appear as doublets, as previously described [83]. B, Northern blot analysis of pre-rRNA intermediates detected with probes specific to the 5'-ETS, the ITS1, and mature $18 \mathrm{~S}$ rRNA (see panel C). C, Processing pathway in Xenopus [38]. Cleavage sites (A' to 5) are 
indicated. The probes used in the northern blotting (panel B) are highlighted in color. (TIF)

S5 Fig. Clustering analysis illustrates the robustness of the RiboMethSeq data. At each of the six developmental stages analyzed, four individual embryos were tested. Clustering of the RiboMethSeq analysis (here shown only for the $18 \mathrm{~S}$ and 5.8S rRNA modifications; the same result was observed with $28 \mathrm{~S}$ rRNA modifications) illustrates the remarkable robustness of our dataset. The staged embryos analyzed are depicted.

S1 Table. List of all the 2'-O methylated positions identified in rRNA by RiboMethSeq in $X$. laevis during development. The table provides the methylation score for each methylated position identified at each stage analyzed. Newly identified positions are highlighted in green. The new positions confirmed by low dNTP primer extension are highlighted in red. The positions modified in human rRNAs are indicated for reference. For simplicity, mean values for the four embryos analyzed at each stage are shown (for deconvoluted data see S2 Table). (XLSX)

S2 Table. Same as S1 Table, with deconvoluted data for each individual embryo analyzed at each stage.

(XLSX)

S3 Table. List of box C/D snoRNA candidates for the identified 2'-O methylated positions in X. laevis. The sequence of box C/D snoRNAs assigned to each 2'-O methylated rRNA position is provided. SnoRNAs which were already present in the Rfam database are indicated by name. C box (blue), D box (green), D' box (purple), and complementary sequences (orange) are highlighted for each newly identified snoRNA. For reference, the positions modified in $X$. tropicalis and the identified snoRNAs in X. tropicalis are indicated (see [67] for details). (XLSX)

S4 Table. List of all the DNA oligonucleotides used in this work. (XLSX)

S5 Table. Sequence of the morpholinos used in this work. (XLSX)

S1 Data. Relative retinal pigmented epithelium (RPE) area data. Primary data used to produce the graph in S2 Fig.

(XLSX)

\section{Acknowledgments}

We thank Sadia Kricha (ULB) for assistance with in vitro transcript synthesis.

\section{Author Contributions}

Conceptualization: Jonathan Delhermite, Lionel Tafforeau, Eric Bellefroid, Denis L. J. Lafontaine.

Data curation: Lionel Tafforeau, Denis L. J. Lafontaine.

Formal analysis: Lionel Tafforeau, Yuri Motorin, Eric Bellefroid, Denis L. J. Lafontaine.

Funding acquisition: Yuri Motorin, Eric Bellefroid, Denis L. J. Lafontaine. 
Investigation: Jonathan Delhermite, Sunny Sharma, Virginie Marchand, Ludivine Wacheul, Ruben Lattuca, Simon Desiderio, Denis L. J. Lafontaine.

Methodology: Denis L. J. Lafontaine.

Project administration: Denis L. J. Lafontaine.

Resources: Denis L. J. Lafontaine.

Supervision: Lionel Tafforeau, Eric Bellefroid, Denis L. J. Lafontaine.

Validation: Lionel Tafforeau, Eric Bellefroid, Denis L. J. Lafontaine.

Visualization: Lionel Tafforeau, Yuri Motorin, Denis L. J. Lafontaine.

Writing - original draft: Lionel Tafforeau, Eric Bellefroid, Denis L. J. Lafontaine.

Writing - review \& editing: Denis L. J. Lafontaine.

\section{References}

1. Steitz TA. A structural understanding of the dynamic ribosome machine. Nature reviews Molecular cell biology. 2008; 9(3):242-53. https://doi.org/10.1038/nrm2352 PMID: 18292779.

2. Henras AK, Plisson-Chastang C, O'Donohue MF, Chakraborty A, Gleizes PE. An overview of pre-ribosomal RNA processing in eukaryotes. Wiley interdisciplinary reviews RNA. 2015; 6(2):225-42. https:// doi.org/10.1002/wrna.1269 PMID: 25346433; PubMed Central PMCID: PMC4361047.

3. Bohnsack KE, Bohnsack MT. Uncovering the assembly pathway of human ribosomes and its emerging links to disease. The EMBO journal. 2019. Epub 2019/05/16. https://doi.org/10.15252/embj. 2018100278 PMID: 31268599

4. Pena C, Hurt E, Panse VG. Eukaryotic ribosome assembly, transport and quality control. Nature structural \& molecular biology. 2017; 24(9):689-99. Epub 2017/09/08. https://doi.org/10.1038/nsmb.3454 PMID: 28880863.

5. Klinge S, Woolford JL, Jr. Ribosome assembly coming into focus. Nature reviews Molecular cell biology. 2019; 20(2):116-31. Epub 2018/11/24. https://doi.org/10.1038/s41580-018-0078-y PMID: 30467428.

6. Lafontaine DL. A 'garbage can' for ribosomes: how eukaryotes degrade their ribosomes. Trends in biochemical sciences. 2010; 35(5):267-77. Epub 2010/01/26. https://doi.org/10.1016/j.tibs.2009.12.006 PMID: 20097077.

7. Genuth NR, Barna M. Heterogeneity and specialized functions of translation machinery: from genes to organisms. Nature reviews Genetics. 2018; 19(7):431-52. Epub 2018/05/05. https://doi.org/10.1038/ s41576-018-0008-z PMID: 29725087.

8. Lafontaine DLJ. Noncoding RNAs in eukaryotic ribosome synthesis and function. Nature Structural and Molecular Biology. 2015; 22(1):11-9. https://doi.org/10.1038/nsmb.2939 PMID: 25565028

9. Henras AK, Plisson-Chastang C, Humbert O, Romeo Y, Henry Y. Synthesis, Function, and Heterogeneity of snoRNA-Guided Posttranscriptional Nucleoside Modifications in Eukaryotic Ribosomal RNAs. Enzymes. 2017; 41:169-213. Epub 2017/06/12. https://doi.org/10.1016/bs.enz.2017.03.007 PMID: 28601222.

10. Sloan KE, Warda AS, Sharma S, Entian KD, Lafontaine DLJ, Bohnsack MT. Tuning the ribosome: the influence of rRNA modification on eukaryotic ribosome biogenesis and function. RNA biology. 2016; 14 (9):1138-52. Epub Epub 2016 Dec 2. https://doi.org/10.1080/15476286.2016.1259781 PMID: 27911188

11. Sharma S, Lafontaine DLJ. "View From A Bridge": A New Perspective on Eukaryotic rRNA base Modification. Trends in biochemical sciences. 2015; 40(10):560-75. https://doi.org/10.1016/j.tibs.2015.07. 008 PMID: 26410597

12. Polikanov $Y S$, Melnikov SV, Soll D, Steitz TA. Structural insights into the role of rRNA modifications in protein synthesis and ribosome assembly. Nature structural \& molecular biology. 2015; 22(4):342-4. https://doi.org/10.1038/nsmb.2992 PMID: 25775268.

13. Higa-Nakamine S, Suzuki T, Uechi T, Chakraborty A, Nakajima $Y$, Nakamura M, et al. Loss of ribosomal RNA modification causes developmental defects in zebrafish. Nucleic Acids Res. 2012; 40(1):391-8. https://doi.org/10.1093/nar/gkr700 PMID: 21908402; PubMed Central PMCID: PMC3245925.

14. Krogh N, Jansson MD, Hafner SJ, Tehler D, Birkedal U, Christensen-Dalsgaard M, et al. Profiling of 2'$\mathrm{O}-\mathrm{Me}$ in human rRNA reveals a subset of fractionally modified positions and provides evidence for 
ribosome heterogeneity. Nucleic Acids Res. 2016; 44(16):7884-95. https://doi.org/10.1093/nar/gkw482 PMID: 27257078; PubMed Central PMCID: PMC27257078.

15. Sharma S, Marchand V, Motorin Y, Lafontaine DLJ. Identification of sites of 2'-O-methylation vulnerability in human ribosomal RNAs by systematic mapping. Scientific reports. 2017; 7(1):11490. https://doi. org/10.1038/s41598-017-09734-9 PMID: 28904332; PubMed Central PMCID: PMC5597630.

16. Erales J, Marchand V, Panthu B, Gillot S, Belin S, Ghayad SE, et al. Evidence for rRNA 2'-O-methylation plasticity: Control of intrinsic translational capabilities of human ribosomes. Proceedings of the National Academy of Sciences of the United States of America. 2017; 114(49):12934-9. https://doi.org/ 10.1073/pnas.1707674114 PMID: 29158377.

17. Taoka M, Nobe Y, Yamaki Y, Sato K, Ishikawa H, Izumikawa K, et al. Landscape of the complete RNA chemical modifications in the human 80 S ribosome. Nucleic Acids Res. 2018; 46(18):9289-98. Epub 2018/09/12. https://doi.org/10.1093/nar/gky811 PMID: 30202881; PubMed Central PMCID: PMC6182160.

18. Ramachandran S, Krogh N, Jorgensen TE, Johansen SD, Nielsen H, Babiak I. The shift from early to late types of ribosomes in zebrafish development involves changes at a subset of rRNA 2'-O-Me sites. RNA. 2020. Epub 2020/09/12. https://doi.org/10.1261/rna.076760.120 PMID: 32912962.

19. Hebras J, Krogh N, Marty V, Nielsen H, Cavaille J. Developmental changes of rRNA ribose methylations in the mouse. RNA biology. 2020; 17(1):150-64. Epub 2019/10/01. https://doi.org/10.1080/15476286. 2019.1670598 PMID: 31566069; PubMed Central PMCID: PMC6948968.

20. Shi Z, Fujii K, Kovary KM, Genuth NR, Rost HL, Teruel MN, et al. Heterogeneous Ribosomes Preferentially Translate Distinct Subpools of mRNAs Genome-wide. Mol Cell. 2017; 67(1):71-83 e7. Epub 2017/ 06/20. https://doi.org/10.1016/j.molcel.2017.05.021 PMID: 28625553; PubMed Central PMCID: PMC5548184.

21. Marcel V, Ghayad SE, Belin S, Therizols G, Morel AP, Solano-Gonzalez E, et al. p53 acts as a safeguard of translational control by regulating fibrillarin and rRNA methylation in cancer. Cancer cell. 2013; 24(3):318-30. Epub 2013/09/14. https://doi.org/10.1016/j.ccr.2013.08.013 PMID: 24029231.

22. Kondrashov N, Pusic A, Stumpf CR, Shimizu K, Hsieh AC, Ishijima J, et al. Ribosome-mediated specificity in Hox mRNA translation and vertebrate tissue patterning. Cell. 2011; 145(3):383-97. Epub 2011/ 05/03. https://doi.org/10.1016/j.cell.2011.03.028 PMID: 21529712; PubMed Central PMCID: PMC4445650.

23. Li D, Wang J. Ribosome heterogeneity in stem cells and development. The Journal of cell biology. 2020; 219(6). Epub 2020/04/25. https://doi.org/10.1083/jcb.202001108 PMID: 32330234; PubMed Central PMCID: PMC7265316.

24. Bellodi C, McMahon M, Contreras A, Juliano D, Kopmar N, Nakamura T, et al. H/ACA small RNA dysfunctions in disease reveal key roles for noncoding RNA modifications in hematopoietic stem cell differentiation. Cell reports. 2013; 3(5):1493-502. https://doi.org/10.1016/j.celrep.2013.04.030 PMID: 23707062; PubMed Central PMCID: PMC3857015.

25. Yamada SB, Gendron TF, Niccoli T, Genuth NR, Grosely R, Shi Y, et al. RPS25 is required for efficient RAN translation of C9orf72 and other neurodegenerative disease-associated nucleotide repeats. Nat Neurosci. 2019; 22(9):1383-8. Epub 2019/07/31. https://doi.org/10.1038/s41593-019-0455-7 PMID: 31358992; PubMed Central PMCID: PMC6713615.

26. Yelick PC, Trainor PA. Ribosomopathies: Global process, tissue specific defects. Rare Dis. 2015; 3(1): e1025185. https://doi.org/10.1080/21675511.2015.1025185 PMID: 26442198; PubMed Central PMCID: PMC4590025.

27. Farley-Barnes KI, Ogawa LM, Baserga SJ. Ribosomopathies: Old Concepts, New Controversies. Trends in genetics: TIG. 2019; 35(10):754-67. Epub 2019/08/05. https://doi.org/10.1016/j.tig.2019.07. 004 PMID: 31376929.

28. Kampen KR, Sulima SO, Vereecke S, De Keersmaecker K. Hallmarks of ribosomopathies. Nucleic Acids Res. 2019; 48(3):1013-28. Epub 2019/07/28. https://doi.org/10.1093/nar/gkz637 PMID: 31350888.

29. Richard EM, Polla DL, Assir MZ, Contreras M, Shahzad M, Khan AA, et al. Bi-allelic Variants in METTL5 Cause Autosomal-Recessive Intellectual Disability and Microcephaly. American journal of human genetics. 2019; 105(4):869-78. Epub 2019/10/01. https://doi.org/10.1016/j.ajhg.2019.09.007 PMID: 31564433; PubMed Central PMCID: PMC6817559.

30. van Tran N, Ernst FGM, Hawley BR, Zorbas C, Ulryck N, Hackert $P$, et al. The human 18S rRNA m6A methyltransferase METTL5 is stabilized by TRMT112. Nucleic Acids Res. 2019; 47(15):7719-33. Epub 2019/07/23. https://doi.org/10.1093/nar/gkz619 PMID: 31328227.

31. Leismann J, Spagnuolo M, Pradhan M, Wacheul L, Vu MA, Musheev M, et al. The 18S ribosomal RNA $\mathrm{m}(6)$ A methyltransferase Mett|5 is required for normal walking behavior in Drosophila. EMBO reports. 2020:e49443. Epub 2020/05/01. https://doi.org/10.15252/embr.201949443 PMID: 32350990. 
32. Uechi T, Nakajima Y, Chakraborty A, Torihara H, Higa S, Kenmochi N. Deficiency of ribosomal protein S19 during early embryogenesis leads to reduction of erythrocytes in a zebrafish model of DiamondBlackfan anemia. Human molecular genetics. 2008; 17(20):3204-11. Epub 2008/07/26. https://doi.org/ 10.1093/hmg/ddn216 PMID: 18653748.

33. Zhao C, Andreeva V, Gibert Y, LaBonty M, Lattanzi V, Prabhudesai S, et al. Tissue specific roles for the ribosome biogenesis factor Wdr43 in zebrafish development. PLoS genetics. 2014; 10(1):e1004074. https://doi.org/10.1371/journal.pgen.1004074 PMID: 24497835; PubMed Central PMCID: PMC3907300.

34. Palasin K, Uechi T, Yoshihama M, Srisowanna N, Choijookhuu N, Hishikawa Y, et al. Abnormal development of zebrafish after knockout and knockdown of ribosomal protein L10a. Scientific reports. 2019; 9(1):18130. Epub 2019/12/04. https://doi.org/10.1038/s41598-019-54544-w PMID: 31792295; PubMed Central PMCID: PMC6889351.

35. Uechi T, Kenmochi N. Zebrafish Models of Diamond-Blackfan Anemia: A Tool for Understanding the Disease Pathogenesis and Drug Discovery. Pharmaceuticals (Basel). 2019; 12(4). Epub 2019/10/12. https://doi.org/10.3390/ph12040151 PMID: 31600948; PubMed Central PMCID: PMC6958429.

36. Gessert S, Maurus D, Rossner A, Kuhl M. Pescadillo is required for Xenopus laevis eye development and neural crest migration. Dev Biol. 2007; 310(1):99-112. Epub 2007/08/31. https://doi.org/10.1016/j. ydbio.2007.07.037 PMID: 17727835.

37. Tecza A, Bugner V, Kuhl M, Kuhl SJ. Pescadillo homologue 1 and Peter Pan function during Xenopus laevis pronephros development. Biol Cell. 2011; 103(10):483-98. Epub 2011/07/21. https://doi.org/10 1042/BC20110032 PMID: 21770895.

38. Griffin JN, Sondalle SB, Del Viso F, Baserga SJ, Khokha MK. The ribosome biogenesis factor Nol11 is required for optimal rDNA transcription and craniofacial development in Xenopus. PLoS genetics. 2015; 11(3):e1005018. Epub 2015/03/11. https://doi.org/10.1371/journal.pgen.1005018 PMID: 25756904; PubMed Central PMCID: PMC4354908.

39. Robson A, Owens ND, Baserga SJ, Khokha MK, Griffin JN. Expression of ribosomopathy genes during Xenopus tropicalis embryogenesis. BMC developmental biology. 2016; 16(1):38. Epub 2016/10/28. https://doi.org/10.1186/s12861-016-0138-5 PMID: 27784267; PubMed Central PMCID: PMC5081970.

40. Griffin JN, Sondalle SB, Robson A, Mis EK, Griffin G, Kulkarni SS, et al. RPSA, a candidate gene for isolated congenital asplenia, is required for pre-rRNA processing and spleen formation in Xenopus. Development. 2018; 145(20). Epub 2018/10/20. https://doi.org/10.1242/dev.166181 PMID: 30337486; PubMed Central PMCID: PMC6215398.

41. Ruggero D, Grisendi S, Piazza F, Rego E, Mari F, Rao PH, et al. Dyskeratosis congenita and cancer in mice deficient in ribosomal RNA modification. Science. 2003; 299(5604):259-62. https://doi.org/10. 1126/science.1079447 PMID: 12522253.

42. Jones NC, Lynn ML, Gaudenz K, Sakai D, Aoto K, Rey JP, et al. Prevention of the neurocristopathy Treacher Collins syndrome through inhibition of p53 function. Nature medicine. 2008; 14(2):125-33. https://doi.org/10.1038/nm1725 PMID: 18246078; PubMed Central PMCID: PMC3093709.

43. LaBonne C, Bronner-Fraser M. Neural crest induction in Xenopus: evidence for a two-signal model. Development. 1998; 125(13):2403-14. Epub 1998/06/04. PMID: 9609823

44. Newton K, Petfalski E, Tollervey D, Caceres JF. Fibrillarin is essential for early development and required for accumulation of an intron-encoded small nucleolar RNA in the mouse. Mol Cell Biol. 2003; 23(23):8519-27. Epub 2003/11/13. https://doi.org/10.1128/MCB.23.23.8519-8527.2003 PMID: 14612397; PubMed Central PMCID: PMC262675.

45. Tollervey D, Lehtonen H, Carmo-Fonseca M, Hurt EC. The small nucleolar RNP protein NOP1 (fibrillarin) is required for pre-rRNA processing in yeast. The EMBO journal. 1991; 10(3):573-83. PubMed Central PMCID: PMC452687. PMID: 1825809

46. Tafforeau L, Zorbas C, Langhendries JL, Mullineux ST, Stamatopoulou V, Mullier R, et al. The complexity of human ribosome biogenesis revealed by systematic nucleolar screening of Pre-rRNA processing factors. Molecular cell. 2013; 51(4):539-51. Epub 2013/08/27. https://doi.org/10.1016/j.molcel.2013.08. 011 PMID: 23973377.

47. Bouffard S, Dambroise E, Brombin A, Lempereur S, Hatin I, Simion M, et al. Fibrillarin is essential for Sphase progression and neuronal differentiation in zebrafish dorsal midbrain and retina. Dev Biol. 2018; 437(1):1-16. Epub 2018/02/27. https://doi.org/10.1016/j.ydbio.2018.02.006 PMID: 29477341.

48. Aittaleb M, Rashid R, Chen Q, Palmer JR, Daniels CJ, Li H. Structure and function of archaeal box C/D sRNP core proteins. Nat Struct Biol. 2003; 10(4):256-63. Epub 2003/02/25. https://doi.org/10.1038/ nsb905 PMID: 12598892.

49. Aittaleb M, Visone T, Fenley MO, Li H. Structural and thermodynamic evidence for a stabilizing role of Nop5p in S-adenosyl-L-methionine binding to fibrillarin. The Journal of biological chemistry. 2004; 279 (40):41822-9. Epub 2004/08/03. https://doi.org/10.1074/jbc.M406209200 PMID: 15286083. 
50. Parlier D, Moers V, Van Campenhout C, Preillon J, Leclere L, Saulnier A, et al. The Xenopus doublesex-related gene Dmrt5 is required for olfactory placode neurogenesis. Dev Biol. 2013; 373(1):39-52. Epub 2012/10/16. https://doi.org/10.1016/j.ydbio.2012.10.003 PMID: 23064029.

51. Savino R, Gerbi SA. In vivo disruption of Xenopus U3 snRNA affects ribosomal RNA processing. The EMBO journal. 1990; 9(7):2299-308. PMID: 2357971; PubMed Central PMCID: PMC551956.

52. Borovjagin AV, Gerbi SA. U3 small nucleolar RNA is essential for cleavage at sites 1,2 and 3 in prerRNA and determines which rRNA processing pathway is taken in Xenopus oocytes. Journal of molecular biology. 1999; 286(5):1347-63. https://doi.org/10.1006/jmbi.1999.2527 PMID: 10064702.

53. Yao RW, Xu G, Wang Y, Shan L, Luan PF, Wang Y, et al. Nascent Pre-rRNA Sorting via Phase Separation Drives the Assembly of Dense Fibrillar Components in the Human Nucleolus. Mol Cell. 2019; 76 (5):767-83. Epub 2019/09/22. https://doi.org/10.1016/j.molcel.2019.08.014 PMID: 31540874.

54. Nicolas E, Parisot $P$, Pinto-Monteiro $C$, de Walque R, De Vleeschouwer $C$, Lafontaine DLJ. Involvement of human ribosomal proteins in nucleolar structure and p53-dependent nucleolar stress. Nature communications. 2016; 7:11390. https://doi.org/10.1038/ncomms11390 PMID: 27265389.

55. Watkins NJ, Bohnsack MT. The box C/D and H/ACA snoRNPs: key players in the modification, processing and the dynamic folding of ribosomal RNA. Wiley interdisciplinary reviews RNA. 2012; 3 (3):397-414. https://doi.org/10.1002/wrna.117 PMID: 22065625.

56. Maden BE. Identification of the locations of the methyl groups in $18 \mathrm{~S}$ ribosomal RNA from Xenopus laevis and man. Journal of molecular biology. 1986; 189(4):681-99. Epub 1986/06/20. https://doi.org/10. 1016/0022-2836(86)90498-5 PMID: 3783688.

57. Maden BE. Locations of methyl groups in $28 \mathrm{~S}$ rRNA of Xenopus laevis and man. Clustering in the conserved core of molecule. Journal of molecular biology. 1988; 201(2):289-314. Epub 1988/05/20. https:// doi.org/10.1016/0022-2836(88)90139-8 PMID: 3418702.

58. Maden BE. The numerous modified nucleotides in eukaryotic ribosomal RNA. Prog Nucleic Acid Res Mol Biol. 1990; 39:241-303. Epub 1990/01/01. https://doi.org/10.1016/s0079-6603(08)60629-7 PMID: 2247610.

59. Birkedal U, Christensen-Dalsgaard M, Krogh N, Sabarinathan R, Gorodkin J, Nielsen H. Profiling of ribose methylations in RNA by high-throughput sequencing. Angew Chem Int Ed. 2014;53. https://doi. org/10.1002/anie.201408362 PMID: 25417815

60. Marchand V, Blanloeil-Oillo F, Helm M, Motorin Y. Illumina-based RiboMethSeq approach for mapping of 2'-O-Me residues in RNA. Nucleic Acids Res. 2016; 44(16):e135. https://doi.org/10.1093/nar/gkw547 PMID: 27302133

61. Maden BE, Corbett ME, Heeney PA, Pugh K, Ajuh PM. Classical and novel approaches to the detection and localization of the numerous modified nucleotides in eukaryotic ribosomal RNA. Biochimie. 1995; 77(1-2):22-9. https://doi.org/10.1016/0300-9084(96)88100-4 PMID: 7599273

62. Bugner V, Tecza A, Gessert S, Kuhl M. Peter Pan functions independently of its role in ribosome biogenesis during early eye and craniofacial cartilage development in Xenopus laevis. Development. 2011; 138(11):2369-78. Epub 2011/05/12. https://doi.org/10.1242/dev.060160 PMID: 21558383.

63. Genuth NR, Barna M. The Discovery of Ribosome Heterogeneity and Its Implications for Gene Regulation and Organismal Life. Mol Cell. 2018; 71(3):364-74. Epub 2018/08/04. https://doi.org/10.1016/j. molcel.2018.07.018 PMID: 30075139; PubMed Central PMCID: PMC6092941.

64. Mills EW, Green R. Ribosomopathies: There's strength in numbers. Science. 2017; 358(6363):1-8. Epub 2017/11/04. https://doi.org/10.1126/science.aan2755 PMID: 29097519.

65. Dixon J, Jones NC, Sandell LL, Jayasinghe SM, Crane J, Rey JP, et al. Tcof1/Treacle is required for neural crest cell formation and proliferation deficiencies that cause craniofacial abnormalities. Proceedings of the National Academy of Sciences of the United States of America. 2006; 103(36):13403-8. https://doi.org/10.1073/pnas.0603730103 PMID: 16938878; PubMed Central PMCID: PMC1557391.

66. Noack Watt KE, Achilleos A, Neben CL, Merrill AE, Trainor PA. The Roles of RNA Polymerase I and III Subunits Polr1c and Polr1d in Craniofacial Development and in Zebrafish Models of Treacher Collins Syndrome. PLoS genetics. 2016; 12(7):e1006187. Epub 2016/07/23. https://doi.org/10.1371/journal. pgen.1006187 PMID: 27448281; PubMed Central PMCID: PMC4957770.

67. Deryusheva S, Talhouarne GJS, Gall JG. "Lost and Found": snoRNA Annotation in the Xenopus Genome and Implications for Evolutionary Studies. Mol Biol Evol. 2020; 37(1):149-66. Epub 2019/09/ 26. https://doi.org/10.1093/molbev/msz209 PMID: 31553476; PubMed Central PMCID: PMC6984369.

68. Sive HLG R, Harland R.M. Early development of Xenopus laevis: a laboratory manual: CSHL Press; 2000.

69. Nieuwkoop PD, Faber J. Normal table of Xenopus laevis (Daudin). Amsterdam, The Netherlands: North Holland; 1967. 
70. Christian JL, McMahon JA, McMahon AP, Moon RT. Xwnt-8, a Xenopus Wnt-1/int-1-related gene responsive to mesoderm-inducing growth factors, may play a role in ventral mesodermal patterning during embryogenesis. Development. 1991; 111(4):1045-55. Epub 1991/04/01. PMID: 1879349.

71. Smith WC, Harland RM. Expression cloning of noggin, a new dorsalizing factor localized to the Spemann organizer in Xenopus embryos. Cell. 1992; 70(5):829-40. Epub 1992/09/04. https://doi.org/10. 1016/0092-8674(92)90316-5 PMID: 1339313.

72. Wilkinson DG, Nieto MA. Detection of messenger RNA by in situ hybridization to tissue sections and whole mounts. Methods in enzymology. 1993; 225:361-73. Epub 1993/01/01. https://doi.org/10.1016/ 0076-6879(93)25025-w PMID: 8231863.

73. Nichane M, de Croze N, Ren X, Souopgui J, Monsoro-Burq AH, Bellefroid EJ. Hairy2-Id3 interactions play an essential role in Xenopus neural crest progenitor specification. Dev Biol. 2008; 322(2):355-67. Epub 2008/08/30. https://doi.org/10.1016/j.ydbio.2008.08.003 PMID: 18721802.

74. Hopwood ND, Pluck A, Gurdon JB. A Xenopus mRNA related to Drosophila twist is expressed in response to induction in the mesoderm and the neural crest. Cell. 1989; 59(5):893-903. Epub 1989/12/ 01. https://doi.org/10.1016/0092-8674(89)90612-0 PMID: 2590945.

75. Schlosser G, Ahrens K. Molecular anatomy of placode development in Xenopus laevis. Dev Biol. 2004; 271(2):439-66. Epub 2004/06/30. https://doi.org/10.1016/j.ydbio.2004.04.013 PMID: 15223346.

76. Thelie A, Desiderio S, Hanotel J, Quigley I, Van Driessche B, Rodari A, et al. Prdm12 specifies V1 interneurons through cross-repressive interactions with Dbx1 and Nkx6 genes in Xenopus. Development. 2015; 142(19):3416-28. Epub 2015/10/08. https://doi.org/10.1242/dev.121871 PMID: 26443638; PubMed Central PMCID: PMC4631751.

77. Hensey C, Gautier J. Programmed cell death during Xenopus development: a spatio-temporal analysis. Dev Biol. 1998; 203(1):36-48. Epub 1998/11/10. https://doi.org/10.1006/dbio.1998.9028 PMID: 9806771

78. Maden BE. Mapping 2'-O-methyl groups in ribosomal RNA. Methods. 2001; 25(3):374-82. https://doi. org/10.1006/meth.2001.1250 PMID: 11860292.

79. Talhouarne GJ, Gall JG. Lariat intronic RNAs in the cytoplasm of Xenopus tropicalis oocytes. RNA. 2014; 20(9):1476-87. Epub 2014/07/24. https://doi.org/10.1261/rna.045781.114 PMID: 25051970; PubMed Central PMCID: PMC4138330.

80. Lowe TM, Eddy SR. A computational screen for methylation guide snoRNAs in yeast. Science. 1999; 283(5405):1168-71. Epub 1999/02/19. https://doi.org/10.1126/science.283.5405.1168 PMID: 10024243.

81. Griffiths-Jones S, Bateman A, Marshall M, Khanna A, Eddy SR. Rfam: an RNA family database. Nucleic Acids Res. 2003; 31(1):439-41. Epub 2003/01/10. https://doi.org/10.1093/nar/gkg006 PMID: 12520045; PubMed Central PMCID: PMC165453.

82. Madeira F, Park YM, Lee J, Buso N, Gur T, Madhusoodanan N, et al. The EMBL-EBI search and sequence analysis tools APIs in 2019. Nucleic Acids Res. 2019; 47(W1):W636-W41. Epub 2019/04/ 13. https://doi.org/10.1093/nar/gkz268 PMID: 30976793; PubMed Central PMCID: PMC6602479.

83. Verheggen C, Le Panse S, Almouzni G, Hernandez-Verdun D. Presence of pre-rRNAs before activation of polymerase I transcription in the building process of nucleoli during early development of Xenopus laevis. The Journal of cell biology. 1998; 142(5):1167-80. Epub 1998/09/10. https://doi.org/10.1083/jcb. 142.5.1167 PMID: 9732279; PubMed Central PMCID: PMC2149348. 